\title{
Nonviral mcDNA-Mediated Cospecific CAR T Cells For The Treatment of Human Hepatocellular Carcinoma Xenograft in Mice
}

Hezhi Wang

Zhongshan Hospital Fudan University https://orcid.org/0000-0002-3194-8086

\section{Xiaoxiao Wang}

Hebei Medical University

\section{Xueshuai Ye}

Hebei Medical University

Yi Ju

Hebei University of Engineering School of Medicine

\section{Nana Cao}

Hebei Normal University College of Life Sciences

\section{Shuqi Wang}

Shijiazhuang traditional Chinese medicine hospital

Jianhui Cai ( $\nabla$ lc_mashikun@tmu.edu.cn )

Hebei Medical University https://orcid.org/0000-0001-6374-4419

\section{Primary research}

Keywords: Hepatocellular carcinoma, Cancer immunotherapy, Nonviral mcDNA vector, Cospecific CAR T cells, Cancer stem cells

Posted Date: November 10th, 2021

DOI: https://doi.org/10.21203/rs.3.rs-1039651/v1

License: (c) (i) This work is licensed under a Creative Commons Attribution 4.0 International License. Read Full License 


\section{Abstract}

Background: Hepatocellular carcinoma (HCC) is one of the most common cancers worldwide and the adoptive immunotherapy of which is worth studying. CD133, a kind of cancer stem cell (CSC) antigen, together with glypican-3 (GPC3) have been proved to be highly expressed in HCC cells and both of them are used as targets to generate chimeric antigen receptor (CAR) T cells. But there are limitations like "ontarget, off-tumor" toxicity, low transfection efficacy and weak antitumor ability in CAR T cells treatment.

Methods: First we fused anti-CD133 and anti-GPC3 single chain Fragment variable (scFv) structures with intracellular domains, respectively. Using non-viral minicircle DNA (mcDNA) vectors to generate cospecific CAR T cells (CoG133-CAR T cells) against CD133 and GPC3 double-positive HCC cells. We exhibited the transduction efficiency of CoG133-CAR T cells and the antigen expression of tumor cell lines. Finally, the antitumor efficacy of CoG133-CAR T cells both in vitro and in vivo was detected.

Results: GPC3-CAR and CD133-CAR were successfully prepared using non-viral mcDNA vectors to generate effector cells. For the GPC3 and CD133 double-positive HCC (Huh7) xenograft mice, co-specific CAR T cells possessed stronger tumor growth suppression compared to single-targeted CAR (GPC3-CAR and CD133-CAR) T cells which induced only one antigen-mediated signal pathway. The same results also occurred on the in vitro experiments including cytokine secretion, cytotoxicity and proliferation ability of CAR T cells. Vital organs from CoG133-CAR T cells and normal T cells respectively treated Huh7 xenograft mice were stained by hematoxylin and eosin (H\&E), the images showed no difference.

Conclusions: The mcDNA vectors loading CAR structures were transfected into T cells by electroporation without genetic mutation or mismatch. Huh7 is an HCC cell line with two antigens of GPC3 and CD133 highly expressed. The antitumor efficacy of co-specific CAR (CoG133-CAR) T cells against Huh7 cells is significantly enhanced. The joint design of two specific targets and non-viral vectors leads much more safety, also.

\section{Introduction}

Primary liver cancer is the sixth most common cancer and the second leading cause of cancer mortality worldwide [1]. Hepatocellular carcinoma (HCC) is the most common type of primary liver cancer, and more than $80 \%$ of cases are associated with the most common risk factor, liver cirrhosis, resulting predominantly from chronic hepatitis $B$ virus (HBV) or hepatitis $\mathrm{C}$ virus (HCV) infection and alcoholic liver disease [2]. Since the overall 5-year survival rate of HCC patients is less than $16 \%$, the development of innovative treatments for $\mathrm{HCC}$ is urgently needed $[3,4]$.

Traditional therapeutic methods, including chemotherapy, radiotherapy, interventional embolization, ablation and surgery, seldom achieve satisfactory effects [5]. These methods are limited by substantial induced suffering, massive cost, iatrogenic metastasis risk and poor prognosis. Immunotherapy with chimeric antigen receptor (CAR)-engineered T cells, which mobilizes internal immunocytes to achieve efficient and painless antitumor outcomes, has been continuously explored and improved. CAR T cells 
first achieved clinical remission (CR) in a B-cell precursor acute lymphoblastic leukemia patient treated with CD19-CAR T cells, and CD19-CAR T cells were approved as a commercial product by the Food and Drug Administration (FDA) for clinical therapy in 2017 [6, 7]. CAR T cell therapy has been nominated by the American Society of Clinical Oncology as the most important advancement in cancer research [8], and its therapeutic efficacy has been proven effective in solid tumors $[9,10]$. We demonstrated that the 3rdgeneration CAR T cells produced by our platform, including PSCA-CAR T cells against prostate cancer and NKG2D-CAR T cells against colorectal cancer, possess significant antitumor capacity both in vitro and in vivo $[11,12]$.

CAR T cells have three components: 1) an extracellular single-chain variable fragment (scFv), which can specifically bind tumor-associated antigens (TAAs) through human leukocyte antigen (HLA)-independent recognition; 2) a hinge domain and transmembrane fragment from human CD8a; and 3) at least one intracellular costimulatory domain such as that from human CD28, CD137 or CD3 3 to promote cell proliferation and the release of cytokines and cytotoxic granules after activation by targeted tumor signals. The method of introducing target sequences into $T$ cells via virus-derived vectors is extensively used. However, this method is often subject to limitations such as safety concerns, low transfection efficacy and considerable cost [13-15]. In addition, the limitations of single antigen-specific CAR T cell treatment, such as "on-target, off-tumor" toxicity and narrow targetability, are difficult to eliminate [16, 17]. To overcome the abovementioned obstacles, we designed CAR T cells based on the progress already achieved by our research team. The newly designed CAR T cells are modified by a nonviral minicircle DNA (mcDNA) vector and featured two scFv structures, thus producing an effective, low-cost and safe treatment.

McDNA vectors are free of bacterial DNA and highly expressed in cells [18]. Glypican-3 (GPC3) is a hallmark of HCC, with the positive expression on 75\% of HCC cells, and CD133 is a marker of cancer stem cells (CSCs) that is also specifically expressed on HCC cells. Both of these antigens induce significant antitumor function in immunotherapy and have been the subject of widespread clinical trials on CARrelated treatments [19-22]. In this study, we transfected GPC3- or CD133-targeting scFv gene sequences into T cells via an mcDNA vector. Then, we harvested the cospecific CAR-engineered T cells (CoG133-CAR T cells), which recognize GPC3 or CD133 to initiate signal transmission. The growth-suppressive effect of CoG133-CAR T cells against double-positive tumors was enormously improved, and the presence of two targeting sites minimized "on-target, off-tumor" toxicity. In summary, this mcDNA-based cospecific CAR T cell system amplified signaling cascade activity in the cell population and exhibited stronger oncolytic activity in terms of cell quality. Moreover, it provides considerable prospects for the development of a new generation of CAR T cells.

\section{Materials And Methods}

\section{Construction of parental plasmid vectors and production of mcDNA}


Based on previous reports, we designed a third-generation GPC3-CAR [23] structure and a secondgeneration CD133-CAR structure $[24,25]$. The DNA sequences of GPC3 scFv and CD133 scFv were derived from monoclonal antibodies (mAbs) described by Nakano et al. [26] and Swaminathan et al. [27]. The GPC3-CAR was composed of the GPC3 ScFv, human CD8a hinge and transmembrane domain (nucleotides 412-609, GenBank NM 001768.6), human CD28 molecule (nucleotides 538-660, GenBank NM 006139.3), human CD137 molecule (nucleotides 640-765, GenBank NM 001561.5) and human CD33 molecule (nucleotides 154-492, GenBank NM 198253.2). The CD133-CAR, containing the CD133 scFv, was linked to the intracellular domains from the human $C D 137$ and $C D 3 \zeta$ molecules via the human CD8a hinge and CD8a transmembrane regions. Ncol and EcoRI sites were incorporated at both ends. We humanized the 2 CAR gene sequences and then synthesized (Detai Biologics, Nanjing, China) and confirmed them by genetic sequencing (Sango Biotech, Shanghai, China). We cloned these 2 CAR structures into pUC57 vectors and then into the parental minicircle plasmid pMC.CMV-Easy ${ }^{\mathrm{TM}}$ (System Biosciences, CA, USA). The pMC.CMV-Easy-GFP-CD133-CAR (8513 bp) parental minicircle plasmid contained the CD133-CAR (1455 bp) and a GFP cassette (758 bp), and pMC.CMV-Easy-GPC3-CAR (7923 bp) contained the GPC3-CAR (1608 bp) without a GFP cassette (to clearly distinguish the constructs in subsequent experiments). We transformed the parental minicircle plasmids into $E$. coli strain ZYCY10P3S2T (System Biosciences), and then added the inducer L- (+)-arabinose (Sigma Chemical, MO, USA) into the bacterial growth medium to mediate recombination between attB and attP. The recombinase $\varphi C 31$ was produced after the recombination and separated the parental minicircle plasmid into mcDNA and the parental bacterial backbone. We extracted the CD133-CAR mcDNA and GPC3-CAR mcDNA with an Endo-Free Plasmid DNA Maxi Kit (Omega Bio-tek, GA, USA) and confirmed them via restriction analysis.

\section{Generation and proliferation of CoG133-CAR T cells}

Peripheral blood mononuclear cells (PBMCs) derived from healthy donors were obtained from the Hebei Blood Center. All donors gave informed consent to use their samples for research purposes. All procedures were performed in accordance with the guidelines approved by Hebei Medical University. PBMCs were isolated with lymphocyte separation medium (Tonbo Biosciences, CA, USA). Primary human CD3+ $T$ cells were negatively selected from PBMCs with MACS CD3 MicroBeads (Miltenyi Biotec, Bergish Gladbach, Germany) and cultured in RPMI-1640 medium (Thermo Fisher Scientific, MA, USA)

supplemented with $10 \%$ heat-inactivated fetal bovine serum (FBS, Thermo Fisher Scientific) at $37^{\circ} \mathrm{C}$ in $5 \%$ C02. Primary T cells were activated with $1000 \mathrm{U} / \mathrm{L} \mathrm{IFN- \gamma}$ (Peprotech, NJ, USA), cultured with $1 \mu \mathrm{g} / \mathrm{ml}$ antiCD3 and anti-CD28 antibodies (Miltenyi Biotec) for 1 day, and then expanded in the presence of $500 \mathrm{U} / \mathrm{ml}$ recombinant human interleukin-2 (IL-2, Peprotech) and $10 \mathrm{U} / \mathrm{ml}$ recombinant human interleukin-15 (IL-15, Peprotech) for 2-5 days. We transfected $5 \times 10^{6} \mathrm{~T}$ cells via electroporation with a 4D-Nucleofector ${ }^{\mathrm{TM}}$ system (Lonza, Cologne, Germany); $3 \mu \mathrm{g}$ of mcDNA control plasmid (System Biosciences), CD133-CAR plasmid or GPC3-CAR plasmid, and 100 $\mu$ of P3 Primary Cell Buffer (Lonza) was added according to the manufacturer's instructions. The EO-115 program was used. CoG133-CAR T cells were generated by simultaneously electroporating $1.5 \mu \mathrm{g}$ of CD133-CAR plasmid and $1.5 \mu \mathrm{g}$ of GPC3-CAR plasmid into T 
cells. The transfected T cells were cultured in fresh medium supplemented with $500 \mathrm{U} / \mathrm{ml}$ IL-2. Fresh medium was added every two days to maintain a concentration of $8 \times 10^{5} \mathrm{cells} / \mathrm{ml}$.

\section{Cell lines and culture conditions}

The human HCC cell lines HepG2 and PLC8024 were obtained from the American Type Culture Collection (ATCC, VA, USA) and cultured in minimal essential medium (MEM, Thermo Fisher Scientific). Huh7 and SK-HEP-1 cells were obtained from the Shanghai Cell Bank (Shanghai, China) and cultured in Dulbecco's modified Eagle's medium (DMEM, Thermo Fisher Scientific). All cell lines were cultured in medium supplemented with 10\% FBS (Thermo Fisher Scientific) and 1\% penicillin-streptomycin (Thermo Fisher Scientific) at $37^{\circ} \mathrm{C}$ in $5 \% \mathrm{CO}_{2}$. For bioluminescence assays, we generated a firefly luciferase expressed Huh7 cell line.

\section{Flow cytometry}

All cell samples were analyzed with a BD FACSCanto ${ }^{\text {TM }}$ flow cytometry system (BD Bioscience, CA, USA), and statistical analysis was conducted in FlowJo software (FlowJo, OR, USA). The phenotype of T cells was assessed with fluorescently labeled antibodies specific for human CD3-PC5, CD4-fluorescein isothiocyanate (FITC) and CD8-FITC, which were obtained from BD Bioscience. Tumor surface antigen expression was detected with antibodies against human CD133- phycoerythrin (PE) (BioLegend, CA, USA) and GPC3-PE (Abcam, MA, USA); isotype control groups were stained with IgG1-PE (Abcam). The expression of GFP in T cells was evaluated in the FITC channel to demonstrate the expression of CD133CAR. The expression of GPC3-CAR was assessed by recombinant biotinylated protein $L$ (Thermo Fisher Scientific) binding PE-conjugated streptavidin (PE-SA, BD Bioscience). All FACS-related cell samples were handled on ice and washed three times with $1 \times$ PBS (Thermo Fisher Scientific) containing 1\% FBS before staining the corresponding antibodies.

\section{In vitro cytotoxicity assays}

Effector cells were cocultured with target cells at increasing effector: target ratios of 1:5, 1:1, 5:1 and 10:1 in flat-bottom 96-well plates (Corning, NY, USA) containing $100 \mu \mathrm{l}$ of T cell culture medium at $37^{\circ} \mathrm{C}$ in $5 \%$ $\mathrm{CO}_{2}$ for 18 hours. Then, we measured the absorbance at $450 \mathrm{~nm}$ according to the Cell Counting Kit-8 instructions (Dojindo Molecular Technologies, Kumamoto, Japan) using an Epoch microplate spectrophotometer (BioTek, VT, USA). We calculated the cytotoxicity of the effector cells with the following formula: specific lysis (\%) = [1- (mixture cell experiment-medium control)/ (target cell spontaneous-medium control)] $\times 100$.

\section{Cytokine secretion assays}

Effector cells were cocultured with target cells in 96-well plates at an effector: target ratio of 5:1 for 24 hours. Supernatants were collected to measure the levels of cytokines, including IL-2, IFN- $y$ and TNF-a, according to the protocols of the enzyme-linked immunosorbent assay (ELISA) kit (Thermo Fisher 
Scientific). Additionally, $5 \times 10^{6}$ effector cells were collected for in vitro experiments, and $100 \mu$ l of peripheral blood was collected from treated xenograft mice for in vivo experiments.

\section{Western blot analysis}

T cells and tumor tissues were lysed with Radioimmunoprecipitation (RIPA) Lysis and Extraction Buffer (Thermo Fisher Scientific) and quantified with a BCA Protein Assay Kit (Thermo Fisher Scientific). Protein lysates were separated on a $12 \%$ sodium dodecyl sulfate-polyacrylamide gel electrophoresis (SDS-PAGE) gel and transferred to a polyvinylidene fluoride (PVDF) membrane (Thermo Fisher Scientific). The PVDF membrane was blocked in AquaBlock Blocking Buffer (EastCoast Bio, ME, USA) for 2 hours, followed by overnight incubation at $4^{\circ} \mathrm{C}$ with the following primary antibodies: anti-CD133 (1:1000, Abcam), antiGPC3 (1:400, Abcam), anti- $\beta$-actin (1:5000, Abcam) and anti-CD3ろ (1:5000, Abcam). Unbound antibodies were washed away with Tris-HCl buffer containing Tween 20, and the PVDF membrane was then incubated with a horseradish peroxidase (HRP)-conjugated secondary antibody (Abcam) for 50 minutes at room temperature. Blots were detected using SuperSignal ${ }^{\mathrm{TM}}$ West Pico PLUS Chemiluminescent Substrate (Thermo Fisher Scientific) and visualized with a ChemiDoc ${ }^{T M}$ Touch Imaging System (BIO-RAD, CA, USA).

\section{Xenograft mouse models}

All animal experiments were conducted in the Clinical Research Center of Hebei General Hospital (HBGH), and all animal procedures were approved by the Animal Care and Management Committee of HBGH. All animal protocols were approved by the Hebei Medical University Animal Care and Use Committee, Hebei, China. Six- to eight-week-old female nonobese diabetic/severe combined immuno-deficiency (NOD/SCID) mice were purchased from Vital River, Beijing, China and were raised in specific pathogen-free (SPF)grade cages and provided autoclaved food and water.

For the subcutaneous HCC models, mice were inoculated subcutaneously with $5 \times 10^{6}$ SK-HEP-1, HepG2, PLC8024 or Huh7 cells on day 0 , and the volumes of tumors derived from these cells were $100 \mathrm{~mm}^{3}$ on day 14 , day 12 , day 17 and day 15 , respectively. Then, the xenograft mice received two intravenous injections of $1 \times 10^{7}$ effector cells on the 3rd and 10th days after the tumor volume reached $100 \mathrm{~mm}^{3}$. For the bioluminescent Huh7 models, mice received $5 \times 10^{6}$ luciferase-labeled Huh7 cells subcutaneously and were then divided randomly into 5 groups $(n=5)$ and injected intravenously with two doses of $1 \times 10^{7}$ effector cells at the abovementioned time points. We measured the tumor volumes and mouse body weights three times weekly, and tumor volumes were calculated with the following formula: $V=1 / 2$ (length $\times$ width $^{2}$ ). Tumor weights were measured after the mice were sacrificed.

\section{Histopathological, immunohistochemical (IHC) and immunofluorescence analyses}

After sacrifice, the Huh7 xenograft mice were perfused with saline and paraformaldehyde at the apex of the heart, and the heart, liver, brain, lung, pancreas, spleen, and intestine were placed in a 
paraformaldehyde fixative for more than 24 hours. All tissues were embedded in paraffin and sliced. Paraffin sections were first dewaxed and stained with hematoxylin. Then, the sections were dehydrated in an alcohol gradient and stained with eosin. Finally, the sections were sealed in neutral gum after dehydration. Three sections were randomly selected from each mouse and photographed under an optical microscope (NIKON, Tokyo, Japan). Paraffin sections of mouse tumors were subjected to the HE staining method described above. After dewaxing, the tumor tissue sections were placed in a repair kit filled with EDTA antigen retrieval buffer $(\mathrm{pH}$ 8.0) for repair. A tissue pen was used to outline the tissue, and an autofluorescence quencher was added. Bovine serum albumin (BSA) was added dropwise in the circle for 30 minutes. For immunohistochemical staining, sections were incubated with anti-CD133 (1:1000, Abcam) and anti-GPC3 (1:200, Abcam) antibodies at $4^{\circ} \mathrm{C}$ overnight and were then washed and incubated with the corresponding secondary antibody at room temperature for 50 minutes. Color development was carried out with 3,3'-diaminobenzidine (DAB), and nuclei were counterstained with hematoxylin. Finally, sections were observed and images were acquired under a microscope. For immunofluorescence staining, sections were incubated with anti-CD133 (1:1000, Abcam), anti-GPC3 (1:200, Abcam) and anti-CD3ろ (1:200, Abcam) antibodies overnight at $4^{\circ} \mathrm{C}$ and then with the corresponding secondary antibody for 50 minutes at room temperature after washing. After incubation with 4',6-diamidino-2-phenylindole (DAPI) for 10 minutes at room temperature, images were acquired under a fluorescence microscope (NIKON, Tokyo, Japan).

\section{Bioluminescence assays}

Cultured Huh7 cells were inoculated bilaterally into the backs of mice to observe tumor growth, and tumors were imaged in vivo when the average volume reached $100 \mathrm{~mm}^{3}$. Ten minutes after subcutaneous injection of $100 \mathrm{mg} / \mathrm{kg}$ D-fluorescein (Solarbio, Beijing, China), mice were anesthetized with isoflurane and were then imaged with a cooled charge-coupled device (CCD) camera system (IVIS

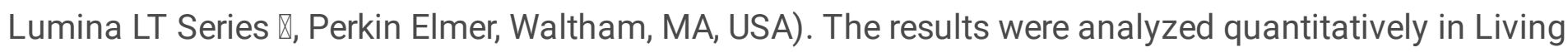
Image software.

\section{Statistical analysis}

Data are presented as the means \pm SDs and were analyzed using Prism 8.0 (GraphPad Software, San Diego, CA). Statistical analysis was carried out using Student's t-test (two-group comparisons), one-way ANOVA with Tukey's post hoc test, and two-way repeated-measures ANOVA followed by Bonferroni's post hoc test. Comparison of survival curves was performed using the log-rank (Mantel-Cox) test. $P<0.05$ was considered statistically significant.

\section{Results}

\section{Construction of the nonviral mcDNA vector and generation of GPC3 and CD133 cospecific CAR-} engineered T cells 
We constructed two nonviral mcDNA vectors encoding GPC3-CAR and CD133-CAR containing the antiGPC3 and anti-CD133 scFv, respectively. We also constructed a third-generation CAR specific to GPC3 and a second-generation CAR specific to CD133. The anti-GPC3 and anti-CD133 scFv were linked to the intracellular domain of the human CD28 or CD137 and CD3 3 molecules via the human CD8a hinge and CD8 a transmembrane regions to enhance costimulatory signaling. To improve the expression efficacy of the chimeric receptor, we added the immunoglobulin kappa light chain (IgG kappa) as the N-terminal leader sequence of anti-GPC3 and anti-CD133 scFv. In addition, to insert the GPC3-CAR and CD133-CAR

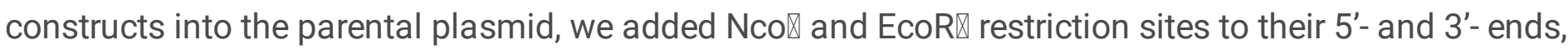
respectively (Figure 1A). We generated two parental minicircle plasmids, pMC.CMV-Easy-GFP-CD133-CAR (8531 bp), with a GFP cassette, and pMC.CMV-Easy-GPC3-CAR (7923 bp), without a GFP cassette (Figure 1C). On gel electrophoresis, six parental minicircle plasmids were shown to be positive clones as detected by double digestion. Lanes 1-3 held pMC.CMV-Easy-GPC3-CAR containing the GPC3-CAR (1608 bp) and lanes 4-6 held pMC.CMV-Easy-GFP-CD133-CAR containing the CD133-CAR (1455 bp) (Figure 1B). Two kinds of mcDNA were generated by $L-(+)$-arabinose-mediated site-specific recombination between attB and attP: CD133-CAR mcDNA (5213 bp) and GPC3-CAR mcDNA (4608 bp) (Figure 1C and 1D). We transfected GPC3 mcDNA and CD133 mcDNA to generate GPC3-CAR T and CD133-CAR T cells, respectively, as control groups and simultaneously transfected GPC3-CAR mcDNA and CD133-CAR mcDNA to generate cospecific CoG133-CAR T cells (Figure 1E).

\section{Expression of CoG133-CAR in human engineered T cells}

We extracted two mcDNA constructs and repeatedly purified them from parental plasmids. GPC3-CAR mcDNA did not encode the GFP gene, and CD133-CAR mcDNA encoded the GFP gene. Then, we simultaneously transfected GPC3-CAR mcDNA and CD133-CAR mcDNA into human T cells via electroporation and generated CoG133-CAR T cells that recognized either the GPC3 or CD133 antigen to induce effector T cell killing of the target tumor cells. Normal T cells without electroporation were established as control cells. Mock T cells were generated by the transfection of the control mcDNA plasmid with the GFP cassette via electroporation; in addition, GPC3-CAR T cells and CD133-CAR T cells were generated by transfection with GPC3-CAR and CD133-CAR mcDNA, respectively, via electroporation.

Significant green fluorescence was observed by fluorescence microscopy in CAR-engineered T cells encoding the GFP gene 6 hours after transfection and constantly increased over the next 24-48 hours (Figure 2A). Seven days after transfection, the expression of CD133-CAR on the cell surface was assessed by determining the GFP expression rate, which was $64.8 \%$ and similar to the GFP expression rate in mock T cells. The expression rate of GPC3-CAR on the cell surface was $65.9 \%$ and was assessed by staining with an antibody against PE-streptavidin (PE-SA) to detect protein L bound to the GPC3 scFv. The expression of CoG133-CAR was 59.1\%, which was assessed by the GPC3/CD133 coexpression rate (Figure 2B). We evaluated the phenotype of normal T cells and CoG133-CAR T cells by CD3/CD4/CD8 labeling and flow cytometric analysis on day 7 . The proportion of $\mathrm{CD}^{+}$cells was approximately $70 \%$, and the ratio of $\mathrm{CD} 4^{+} / \mathrm{CD} 8^{+}$cells was close to $2: 1$, consistent with the human $\mathrm{T}$ cell phenotype under physiological conditions. These results indicated no significant difference in the proportion of $\mathrm{CD}^{+}, \mathrm{CD}^{+}$ 
and $\mathrm{CD} 8^{+}$cells between the two groups (Figures $2 \mathrm{C}$ and 2D). In addition, we used RIPA lysis buffer to extract three kinds of CAR proteins from CAR-engineered T cells, and we then incubated them with an anti-CD3 3 mAb to determine whether GPC3-CAR and CD133-CAR were successfully transfected. In contrast to GPC3-CAR T cells and CD133-CAR T cells, which expressed only a single exogenous CD3ろ protein, CoG133-CAR T cells simultaneously expressed GPC3-scFv-CD28-CD137-CD33(58kDa) and CD133-scFv-CD137-CD3Z(53kDa) fusion proteins, as demonstrated by the successful detection of double exogenous $\mathrm{CD} 3 \zeta$ expression. The expression of endogenous $\mathrm{CD} 3 \zeta$ protein in T cells was detected in all groups of T cells (Figure 2E).

\section{Expression profiles of CD133 and GPC3 in human HCC cell lines and tissues}

We stained four types of human HCC cells with PE-conjugated anti-CD133 and PE-conjugated anti-GPC3 mAbs to examine the expression of the CD133 and GPC3 antigens and stained isotype control groups of 4 tumors with a PE-conjugated anti-IgG1 mAb. The expression rates of the CD133 and GPC3 antigens were $0.1 \%$ and $0.4 \%$, respectively, in SK-HEP- 1 cells; $3.1 \%$ and $80.1 \%$, respectively, in HepG2 cells; $78.8 \%$ and $2.7 \%$, respectively, in PLC8024 cells; and $82.4 \%$ and $98.5 \%$, respectively, in Huh7 cells. We concluded that SK-HEP-1 cells were negative for both antigens, HepG2 cells were positive for the GPC3 antigen, PLC8024 cells were positive for the CD133 antigen and Huh7 cells were positive for both antigens (Figure $3 \mathrm{~A})$. To further demonstrate that the expression of tumor antigens was consistent after the injection of these four human HCC cell lines into NOD/SCID mice and successful modeling, we subjected tumor cells to immunohistochemical staining, and the tumor antigen expression outcomes are shown in electron micrographs. HepG2 cells were positively stained with the anti-GPC3 mAb, PLC8024 cells were positively stained with the anti-CD133 mAb, Huh7 cells were positively stained with both antibodies and SK-HEP-1 cells were negative for both antibodies (Figure 3B). The intensity of CD133 and GPC3 staining in human HCC cells was determined by semiquantitative integrated optical density (IOD) analysis. CD133 antigens were significantly increased in PLC8024 cells and Huh7 cells; GPC3 antigens were significantly increased in HepG2 cells and Huh7 cells (Figure 3C). We further confirmed the expression of GPC3 and CD133 proteins in human HCC tissues by Western blot analysis. GPC3 protein was expressed in the Huh7 and HepG2 cell lines, and CD133 protein was expressed in the Huh7 and PLC8024 cell lines (Figure 3D). After verifying the tumor expression profiles by the above methods, we concluded that the SK-HEP-1 cell line was GPC3 ${ }^{-}$and $\mathrm{CD}^{-133^{-}}$, the HepG2 cell line was $\mathrm{GPC}^{+}$and $\mathrm{CD}^{-133^{-}}$, the PLC8024 cell line was $\mathrm{GPC}^{+}$and $\mathrm{CD}_{133}$, and the Huh7 cell line was $\mathrm{GPC}^{+}$and $\mathrm{CD} 133^{+}$.

\section{Cytotoxicity of CoG133-CAR T cells in vitro}

In vitro cytotoxic activity was assessed after overnight coincubation of effector and target cells at distinct ratios. Single-target CAR-engineered T cells such as GPC3-CAR T and CD133-CAR T cells efficiently lysed single-positive tumor cells, and dual-target CAR-engineered T cells such as CoG133-CAR T cells had similar lysis rates against single-positive tumor cells (Figure 4A). Notably, the lysis rate of double-positive tumor cells showed that the cytotoxic activity of CoG133-CAR T cells was significantly enhanced compared with that of GPC3-CAR T cells and CD133-CAR T cells (Figure 4A). We used a double-negative 
SK-HEP-1 cell line as a target cell control group and normal T and mock T cells as effector control groups. The statistical results indicated that the cytotoxic activity was positively correlated with the targetdependent specificity of CAR-engineered T cells.

\section{Cytokine secretion and proliferation abilities of CoG133-CAR T cells}

The intracellular domain of our designed CAR structure originated from $\mathrm{CD} 3 \zeta$ and costimulatory signals, and cytokine secretion was enhanced through the resulting synergism. The cytokines secreted by CARengineered T cells coincubated with corresponding single-positive tumor cells were increased. Moreover, through interaction with double-positive tumor cells, CoG133-CAR T cells were stimulated to secrete greatly increased levels of cytokines (Figure 4B). We assessed the proliferative ability of CAR-engineered T cells stimulated by human HCC cells weekly without the addition of exogenous cytokines. The amount of $T$ cells without a CAR structure (normal $T$ and mock $T$ cells) increased moderately at approximately 14 days and decreased after 2 weeks, similar to the effects of coincubation with the 4 tumor cell lines. Single-and double-target CAR T cells coincubated with single-positive tumor cells showed the same proliferative ability; the proliferation of both began to increase after 7 days and expanded approximately 20- to 25-fold after 28 days. After coincubation with the double-positive Huh7 cell line, single-target CAR T cells expanded 20 - to 25 -fold as above, while dual-target CAR T cells expanded substantially 40 -fold (Figure 4C). We concluded from these statistics that the cytokine secretion and the number of CoG133CAR T cells were significantly increased by the coincubation with the double-positive tumor cell line.

\section{Compared with single-target CAR T cells, CoG133-CAR T cells showed significantly improved antitumor activity in vivo against HCC xenografts}

Our in vitro results indicated that CoG133-CAR T cells were activated by $\mathrm{GPC}^{+} \mathrm{CD} 133^{+}$tumor cells and exhibited vigorous antitumor activity against double-positive cell lines. To detect the in vivo efficacy of CoG133-CAR T cells against HCC, we established HCC xenograft mouse models by inoculating $1 \times 10^{6}$ tumor cells from four kinds of cell lines into NOD/SCID mice. We treated the xenograft mice with $1 \times 10^{7}$ effector cells on day 3 and day 10 after tumor formation and then sacrificed them on day 29. Next, we simultaneously detected tumor antigen expression and $\mathrm{T}$ cell infiltration by immunofluorescence imaging. GPC3 or CD133 single-positive tumors exhibited a small amount of CD3 white fluorescence after CoG133-CAR T cell treatment. More importantly, Huh7 tumor tissue treated with CoG133-CAR T cells showed a large amount of fluorescence indicating T cell infiltration; in addition, strong positive GPC3 and CD133 fluorescence was simultaneously observed (Figure 5A). We measured the volume of tumors from xenograft mice and the weight of tumor tissues after the mice were sacrificed (Figures 5B and 5C). The tumor size in HepG2 and PLC8024 xenograft mice treated with CoG133-CAR T cells were decreased and did not significantly differ from those in xenograft mice injected with GPC3-CAR T or CD133-CAR T cells. However, the tumor sizes in Huh7 xenograft mice treated with CoG133-CAR T cells were significantly reduced. In vivo cytokine secretion, as detected by ELISA on day 7 showed that the levels of IL-2, IFN-Y and TNF-a in blood serum of GPC3 ${ }^{+} \mathrm{CD}_{13} 3^{+}$xenograft mice were the highest among the groups (Figure 5D). These results indicated that $T$ cells were strongly activated only when CoG133-CAR T cells received 
integrated costimulatory signals from both the GPC3-targeting antigen and the CD133-targeting antigen, while the activation signals obtained in single-positive tumor-bearing mice were minimal.

\section{CoG133-CAR T cells exhibited significant growth suppression efficacy in GPC3+CD133+ tumor xenograft mice}

To detect the in vivo suppression efficacy of dual-targeted CAR T cells on GPC $3^{+} \mathrm{CD} 133^{+}$tumor cells, we established Huh7-NOD/SCID xenograft mouse models by subcutaneously inoculating $1 \times 10^{6}$ Huh7 cells into their dorsal regions on day 0 . Subsequently, we randomly divided the mice into 5 groups on day 7 . The tumors in xenograft mice grew to $100 \mathrm{~mm}^{3}$ on day 14 , and bioluminescence images were acquired after the first injection of $1 \times 10^{7}$ effector cells into the mice. We performed a second injection of $1 \times 10^{7}$ effector cells in the mice on day 21 and acquired bioluminescence images on days 28 and 42 (Figure 6A). The tumor burden was assessed by bioluminescence imaging and revealed that CoG133-CAR T cells induced tumor growth suppression in mice. The tumors disappeared in two of five (40\%) xenograft mice, and the other three mice achieved obvious relief by CoG133-CAR T cell injection compared with mice injected with the other effector cells, which exhibited no antitumor efficacy (Figure 6B). The bioluminescence imaging results were statistically analyzed in a region of interest (ROI) (Figure 6C). Ultimately, CoG133-CAR T cells induced a significant survival advantage $(n=5, P<0.001)$ (Figure 6D). In summary, these experimental results indicated that dual-targeted CAR T cells had a potent ability to eradicate Huh7 tumor cells in vivo and prolonged the survival time of Huh7 xenograft mice.

\section{Detection of the persistence and "on-target, off-tumor" toxicities of CoG133-CAR T cells in vivo}

We detected the phenotypes and the CAR expression of CoG133-CAR T cells from the peripheral blood of Huh7 xenograft mice by flow cytometric analysis on day 7 after the second injection of CoG133-CAR T cells. Normal T cells were used as negative control groups. The proportion of $\mathrm{CD} 4^{+}$cells in CoG133-CAR T cells from peripheral blood of mice was significantly reduced compared with that before treatment, but the proportion of $\mathrm{CD} 4^{+}$cells in normal T cells did not significantly differ from that before treatment (Figures 2C, 2D, 7A and 7B). More importantly, the CoG133-CAR expression rate was around $40 \%$ (Figures 7C and 7D). We excised murine organs from sacrificed tumor-bearing mice treated with effector cells and stained them with hematoxylin and eosin (H\&E). Histopathological analysis showed no change in the organs from effector cell-treated mice compared to those from untreated mice. The images from Huh7 tumor-bearing mice treated with CoG133-CAR T cells were exhibited only (Figure 7E).

\section{Discussion}

Immunotherapy has shown broad prospects in cancer treatment [28]. Immune checkpoint inhibitors (antiPD-1/PD-L1 and anti-CTLA-4 mAbs), dendritic cell (DC)-based vaccines, cytokine-induced killer (CIK) cells, cytotoxic T lymphocytes (CTLs) and CAR-engineered T cells are approved as monotherapies or combination treatments for different types of cancer [29]. In this study, we focused on CAR T cells 
because of their genetic modification ability, inherent cytotoxic nature and antigen identify characteristic. Our design integrates an mcDNA vector and dual-targeted CAR T cells.

Compared with conventional plasmids, mcDNA is a miniature nonviral DNA vector without a deleterious bacterial backbone that performs well in both gene transfection efficiency and biological safety [30]. We constructed a second-generation CAR structure encoding the CD133 scFv (containing a GFP cassette) and a third-generation CAR structure encoding the GPC3 ScFv (excluding a GFP cassette) in parental plasmids. Then, two types of mcDNA vectors were recombined from parental plasmids and simultaneously transfected into primary $T$ cells via electroporation to generate CoG133-CAR T cells. The sizes of the GPC3-CAR mcDNA and CD133-CAR mcDNA were $4608 \mathrm{bp}$ and $5213 \mathrm{bp}$, respectively (Figures $1 \mathrm{c}$ and $1 \mathrm{~d}$ ), within the optimal loading range (3000bp-6000bp) of mcDNA vectors. Therefore, the simultaneous transfection of two mcDNA-CAR structures into T cells is theoretically reasonable. To illustrate the excellent gene transfection efficiency of the mcDNA vector, strategies to detect CAR expression were adopted, and the CARs exhibited similar expression proportions in both single-specific and cospecific CAR T cells (Figure 2B).

GPC3 belongs to the heparan sulfate proteoglycan family and is classified as an oncofetal glycoprotein [31]. As an ideal target for adoptive immunotherapy for HCC, GPC3 is overexpressed on the membrane of carcinomatous hepatocytes and negatively detected in normal human tissues and organs [32, 33]. CD133 was first extracted from CD34+ hematopoietic stem cells using an anti-AC133 mAb and is specifically expressed in several cancers, including HCC $[34,35]$. CD133 is a generally confirmed marker of CSCs with significant impacts on the signal transduction and regulation, proliferation, recurrence and drug resistance of tumors $[36,37]$. The expression rates of CD133 and GPC3 in Huh7 cells we confirmed were $82.4 \%$ and $98.5 \%$, respectively (Figure $3 \mathrm{~A}$ ). Differ from the bispecific CAR T cells which contain two targeted antigen-binding sites on one ScFv and activate signal transduction pathway only when both TAAs are recognized, our design of cospecific CAR T cells killed tumor cells by binding one of both antigens. The signal activation ability of ScFv structures with simultaneous binding to two antigens is much weaker than that of ScFv structures with independent binding to two antigens. The CoG133-CAR T cells applied in the present study had two independent scFv antigen-binding sites after GPC3 and CD133 gene sequences were transfected into T cells via mcDNA vectors were generated after the GPC3 and CD133 gene sequences were simultaneously transfected into T cells via mcDNA vectors. Importantly, if we found two antigens highly expressed on a particular type of HCC cell, the corresponding cospecific CAR T cells could exhibit suppression capacity on this HCC cell.

An important reason that malignant tumors are difficult to eradicate is tumor heterogeneity. Tumor heterogeneity originates from the inhomogeneity of the external environment and the randomness of gene mutations and results in the type diversity of cells in the same tumor [38]. CSCs play a critical role in tumor heterogeneity and trigger the self-renewal, multilineage division and sustained growth of tumor cells [39]. As mentioned previously, CD133+ tumor cells belong to CSCs, and CD133 antigen is highly expressed in the PLC8024 and Huh7 cell lines that we selected. CD133 localizes to cellular protrusions and guides CD133-targeted T cells to enter CD133 ${ }^{+}$tumor cells for CSCs elimination [40]. Very 
importantly, these tumor-killing effects overcome the limitation imposed by the tumor microenvironment (TME) by the release of cytokines and CTLs inside the tumor. Two transformations occur in oncology: CSCs can transform into normal cancer cells, and CSCs can transform into normal cells. Both transformations are regulated by the TME [41]. CD133 is a main marker of CSCs with strong cell penetration and GPC3 is a superior HCC marker with high expression [32]. Since GPC3 antigens are widely present on the surface of HCC cells, the GPC3 branch interacted considerably with $\mathrm{GPC}^{+} \mathrm{HCC}$ cells to anchor CoG133-CAR T cells at the tumor and accumulate effector cells around the tumor. The CD133 branch bound CSC-positive HCC cells and activated the intracellular domains of the CAR structures including the CD137 costimulatory molecule and the CD3Z immunoreceptor tyrosine-based activation motif (ITAM). Then, tumor proliferation and differentiation were inhibited. GPC3 provided extensive adhesion for CoG133-CAR T cells, and CD133 provided profound tumor suppression. Both of these antigens were used in our study to counteract the restrictive effects of the TME.

"On-target, off-tumor" toxicity is mainly caused by the expression of TAAs in normal tissues. If major human organs express TAAs and are degraded by CAR T cells, life-threatening adverse effects occur in the human body [42]. In this study, T cells were genetically engineered into cospecific CAR T cells containing two tumor antigen-binding sites (CD133 and GPC3) and were used to treat patients with double-positive HCC. The design of corresponding combined sites existed in both effector and target cells, evidently alleviating the "on-target, off-tumor" toxicity, an effect that has been proven in many previous studies [43]. We evaluated vital organs from CoG133-CAR T cell-treated and normal T celltreated mice via H\&E staining. The histopathological images showed that no obvious difference in any vital organ from CoG133-CAR T cell-treated mice (Figure 7C). Moreover, CoG133-CAR T cells did not have toxicity to normal tissues on mice models.

\section{Conclusion}

Our study indicated that the strategy for mcDNA-mediated CAR-T cells production resulted in excellent transfection efficacy while preventing virus-related adverse effects. The cospecific CAR T cells induced a higher quantity of effector cells to act on double-positive HCC cells and exhibited a CSC-related antitumor ability to destroy the TME. Our work also demonstrated that the design of parallel-connected scFv structures on CoG133-CAR T cells provided precise recognition both in vitro and in vivo. The prolonged survival and tumor reduction were seen in Huh7 xenograft mice treated with CoG133-CAR T cells highlighted the immense prospects of mcDNA vectors and cospecific CAR T cells.

\section{Abbreviations}

HCC, Hepatocellular carcinoma; CSC, cancer stem cell; GPC3, glypican-3; CAR, chimeric antigen receptor; mc, minicircle; $H B V$, hepatitis $B$ virus; $H C V$, hepatitis $C$ virus; $C R$, clinical remission; scFv, single-chain variable fragment; TAA, tumor-associated antigen; HLA, human leukocyte antigen; PBMC, Peripheral blood mononuclear cell; DC, dendritic cell; CIK, cytokine-induced killer; CTL, cytotoxic T lymphocyte; TME tumor microenvironment ; ITAM, immunoreceptor tyrosine-based activation motif. 


\section{Declarations}

\section{Availability of data and materials}

The datasets used or analysed during the current study are available from the corresponding author on reasonable request.

All data generated or analysed during this study are included in this published article and its supplementary information files.

\section{Competing interests}

The authors declare that they have no competing interests.

\section{Funding}

This work was supported by the Graduate Innovation Fund of Hebei (grant numbers CXZZBS2019120.

\section{Authors' contribution}

HZW and XXW performed the most experiments, analyzed data and wrote the paper. XSY established animal models. YJ and NNC performed plasmid construction. SQW participated in data collection and analysis.

$\mathrm{JHC}$ engaged in project design and manuscript revision. All authors approved the final version of the manuscript. JHC was responsible for the integrity of the data and the accuracy of the data analysis.

\section{Acknowledgements}

The authors wish to thank Prof. Yi Guo (College of Life Sciences, Hebei Normal University) for technical support, Prof. Yunli Yan (Department of Cell Biology, Hebei Medical University) and Dr. Yanling Wang (Department of Cell Biology, Hebei Medical University) for facility support.

\section{Authors' information}

${ }^{1}$ Department of Liver Surgery and Transplantation, Liver Cancer Institute, Zhongshan Hospital, Fudan University; Key Laboratory of Carcinogenesis and Cancer Invasion of Ministry of Education, 180 Fenglin Road, Shanghai 200032, China

${ }^{2}$ Department of Surgery, Hebei Medical University, 361 West Zhongshan Road, Shijiazhuang 050000, China

${ }^{3}$ Department of Surgery \& Oncology, Hebei General Hospital, 348 West Heping Road, Shijiazhuang 050000, China 
${ }^{4}$ Department of Internal Medicine, Hebei Medical University, 361 West Zhongshan Road, Shijiazhuang 050000, China

${ }^{5}$ Department of Medicine, Medical College of Hebei University of Engineering, 10 Taiji Road, Handan 056002, China

${ }^{6}$ College of Life Sciences, Hebei Normal University, 20 East 2nd Ring South Road, Shijiazhuang 050000, China

${ }^{7}$ Department of Anorectal Surgery, Shijiazhuang traditional Chinese medicine hospital, 233 West Zhongshan Road, Shijiazhuang 050000, China

\section{References}

1. Bray F, Ferlay J, Soerjomataram I, Siegel RL, Torre LA, Jemal A. Global cancer statistics 2018: GLOBOCAN estimates of incidence and mortality worldwide for 36 cancers in 185 countries. CA: a cancer journal for clinicians. Nov 2018;68(6):394-424.

2. Dawkins J, Webster RM. The hepatocellular carcinoma market. Nature reviews. Drug discovery. Jan 2019;18(1):13-14.

3. European Association for the Study of the Liver. EASL Clinical Practice Guidelines: Management of hepatocellular carcinoma. J Hepatol. Jul 2018;69(1):182-236.

4. Villanueva A. Hepatocellular Carcinoma. The New England journal of medicine. Apr 11 2019;380(15):1450-1462.

5. Song P, Cai Y, Tang H, Li C, Huang J. The clinical management of hepatocellular carcinoma worldwide: A concise review and comparison of current guidelines from 2001 to 2017. Bioscience trends. 2017;11(4):389-398.

6. June $\mathrm{CH}, \mathrm{O}$ 'Connor RS, Kawalekar OU, Ghassemi S. CAR T cell immunotherapy for human cancer. Science. Mar 23 2018;359(6382):1361-1365.

7. Neelapu SS, Locke FL, Bartlett NL, et al. Axicabtagene Ciloleucel CAR T-Cell Therapy in Refractory Large B-Cell Lymphoma. The New England journal of medicine. Dec 28 2017;377(26):2531-2544.

8. Heymach J, Krilov L, Alberg A, et al. Clinical Cancer Advances 2018: Annual Report on Progress Against Cancer From the American Society of Clinical Oncology. Journal of clinical oncology: official journal of the American Society of Clinical Oncology. Apr 1 2018;36(10):1020-1044.

9. Lv J, Zhao R, Wu D, et al. Mesothelin is a target of chimeric antigen receptor T cells for treating gastric cancer. J Hematol Oncol. Feb 18 2019;12(1):18.

10. Brown CE, Alizadeh D, Starr R, et al. Regression of Glioblastoma after Chimeric Antigen Receptor TCell Therapy. The New England journal of medicine. Dec 29 2016;375(26):2561-2569.

11. Han J, Gao F, Geng S, et al. Minicircle DNA-Engineered CAR T Cells Suppressed Tumor Growth in Mice. Mol Cancer Ther. Jan 2020;19(1):178-186. 
12. Deng X, Gao F, Li N, et al. Antitumor activity of NKG2D CAR-T cells against human colorectal cancer cells in vitro and in vivo. Am J Cancer Res. 2019;9(5):945-958.

13. Long AH, Haso WM, Shern JF, et al. 4-1BB costimulation ameliorates $T$ cell exhaustion induced by tonic signaling of chimeric antigen receptors. Nat Med. Jun 2015;21(6):581-590.

14. Nafissi N, Slavcev R. Construction and characterization of an in-vivo linear covalently closed DNA vector production system. Microbial cell factories. Dec 6 2012; 11:154.

15. Cheng C, Tang N, Li J, et al. Bacteria-free minicircle DNA system to generate integration-free CAR-T cells. Journal of medical genetics. Jan 2019;56(1):10-17.

16. Morgan RA, Yang JC, Kitano M, Dudley ME, Laurencot CM, Rosenberg SA. Case report of a serious adverse event following the administration of $T$ cells transduced with a chimeric antigen receptor recognizing ERBB2. Molecular therapy: the journal of the American Society of Gene Therapy. Apr 2010;18(4):843-851.

17. Neelapu SS, Tummala S, Kebriaei P, et al. Chimeric antigen receptor T-cell therapy - assessment and management of toxicities. Nature reviews. Clinical oncology. Jan 2018;15(1):47-62.

18. Jia F, Wilson KD, Sun $\mathrm{N}$, et al. A nonviral minicircle vector for deriving human iPS cells. Nature methods. Mar 2010;7(3):197-199.

19. Flecken $\mathrm{T}$, Schmidt $\mathrm{N}$, Hild $\mathrm{S}$, et al. Immunodominance and functional alterations of tumorassociated antigen-specific CD8+ T-cell responses in hepatocellular carcinoma. Hepatology. Apr 2014;59(4):1415-1426.

20. Gao H, Li K, Tu H, et al. Development of T cells redirected to glypican-3 for the treatment of hepatocellular carcinoma. Clinical cancer research: an official journal of the American Association for Cancer Research. Dec 15 2014;20(24):6418-6428.

21. Miranda-Lorenzo I, Dorado J, Lonardo E, et al. Intracellular autofluorescence: a biomarker for epithelial cancer stem cells. Nature methods. Nov 2014;11(11):1161-1169.

22. Wang $Y$, Chen M, Wu Z, et al. CD133-directed CAR T cells for advanced metastasis malignancies: A phase I trial. Oncoimmunology. 2018;7(7):e1440169.

23. Li W, Guo L, Rathi P, et al. Redirecting T Cells to Glypican-3 with 4-1BB Zeta Chimeric Antigen Receptors Results in Th1 Polarization and Potent Antitumor Activity. Human gene therapy. May 2017;28(5):437-448.

24. Feng KC, Guo YL, Liu Y, et al. Cocktail treatment with EGFR-specific and CD133-specific chimeric antigen receptor-modified $\mathrm{T}$ cells in a patient with advanced cholangiocarcinoma. $\mathrm{J}$ Hematol Oncol. Jan 5 2017;10(1):4.

25. Klapdor R, Wang S, Hacker U, et al. Improved Killing of Ovarian Cancer Stem Cells by Combining a Novel Chimeric Antigen Receptor-Based Immunotherapy and Chemotherapy. Human gene therapy. Oct 2017;28(10):886-896.

26. Nakano K, Orita T, Nezu J, et al. Anti-glypican 3 antibodies cause ADCC against human hepatocellular carcinoma cells. Biochemical and biophysical research communications. Jan 9 2009;378(2):279-284. 
27. Swaminathan SK, Olin MR, Forster CL, Cruz KS, Panyam J, Ohlfest JR. Identification of a novel monoclonal antibody recognizing CD133. Journal of immunological methods. Sep 30 2010;361(12):110-115.

28. Demaria $\mathrm{O}$, Cornen $S$, Daëron $M$, Morel $Y$, Medzhitov R, Vivier E. Harnessing innate immunity in cancer therapy. Nature. Oct 2019;574(7776):45-56.

29. Bailey SR, Maus MV. Gene editing for immune cell therapies. Nat Biotechnol. Dec 2019;37(12):14251434.

30. Zhao J, Huang P, Wang Z, et al. Synthesis of Amphiphilic Poly ( $\beta$-amino ester) for Efficiently Minicircle DNA Delivery in Vivo. ACS Appl Mater Interfaces. Aug 3 2016;8(30):19284-19290.

31. Filmus J, Capurro M, Rast J. Glypicans. Genome biology. 2008;9(5):224.

32. Nishida T, Kataoka H. Glypican 3-Targeted Therapy in Hepatocellular Carcinoma. Cancers (Basel). Sep 10 2019;11(9).

33. Pellegrini M, Pilia G, Pantano S, et al. Gpc3 expression correlates with the phenotype of the SimpsonGolabi-Behmel syndrome. Developmental dynamics: an official publication of the American Association of Anatomists. Dec 1998;213(4):431-439.

34. Yin AH, Miraglia S, Zanjani ED, et al. AC133, a novel marker for human hematopoietic stem and progenitor cells. Blood. Dec 15 1997;90(12):5002-5012.

35. Castelli G, Pelosi E, Testa U. Liver Cancer: Molecular Characterization, Clonal Evolution and Cancer Stem Cells. Cancers. Sep 20 2017;9(9).

36. Matteucci C, Balestrieri E, Argaw-Denboba A, Sinibaldi-Vallebona P. Human endogenous retroviruses role in cancer cell stemness. Seminars in cancer biology. Dec 2018;53:17-30.

37. Zeki SS, Graham TA, Wright NA. Stem cells and their implications for colorectal cancer. Nature reviews. Gastroenterology \& hepatology. Feb 2011;8(2):90-100.

38. Heppner GH, Shekhar M. Tumor heterogeneity is fundamental to the tumor ecosystem. Oncology (Williston Park, N.Y.). Sep 2014;28(9):780-781.

39. Zöller M. CD44: can a cancer-initiating cell profit from an abundantly expressed molecule? Nat Rev Cancer. Apr 2011;11(4):254-267.

40. Aghajani M, Mansoori B. New emerging roles of CD133 in cancer stem cell: Signaling pathway and miRNA regulation. J Cell Physiol. Dec 2019;234(12):21642-21661.

41. Najafı M, Farhood B, Mortezaee K. Cancer stem cells (CSCs) in cancer progression and therapy. J Cell Physiol. Jun 2019;234(6):8381-8395.

42. Akhavan D, Alizadeh D, Wang D, Weist MR, Shepphird JK, Brown CE. CAR T cells for brain tumors: Lessons learned and road ahead. Immunol Rev. Jul 2019;290(1):60-84.

43. Yu S, Yi M, Qin S, Wu K. Next generation chimeric antigen receptor T cells: safety strategies to overcome toxicity. Mol Cancer. Aug 20 2019;18(1):125.

\section{Figures}


A

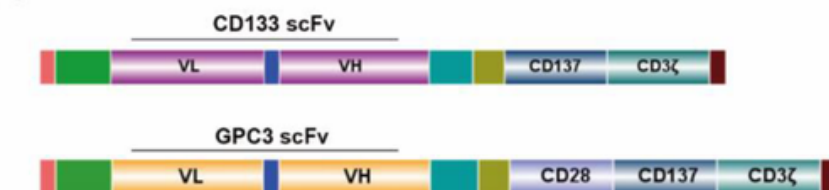

B

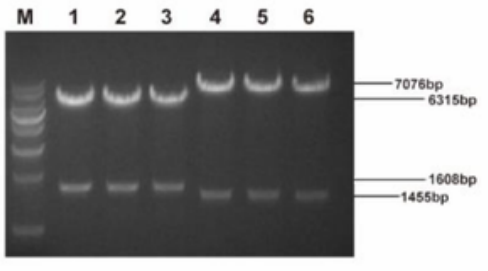

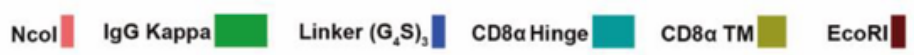

C
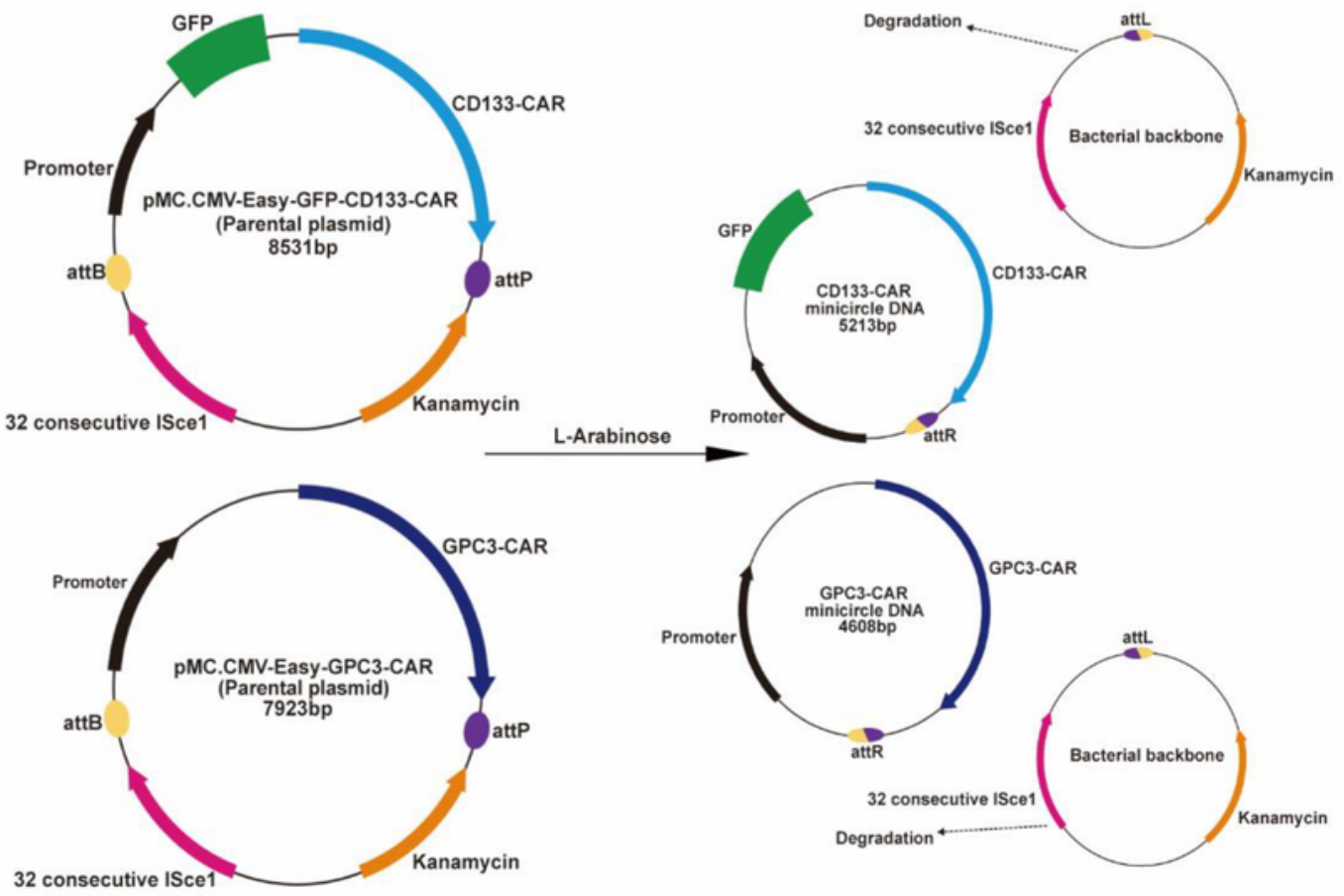

D

$\mathbf{E}$

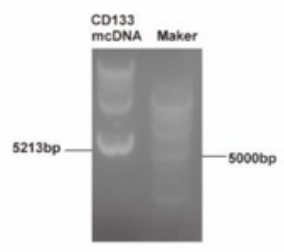

Eancer cell
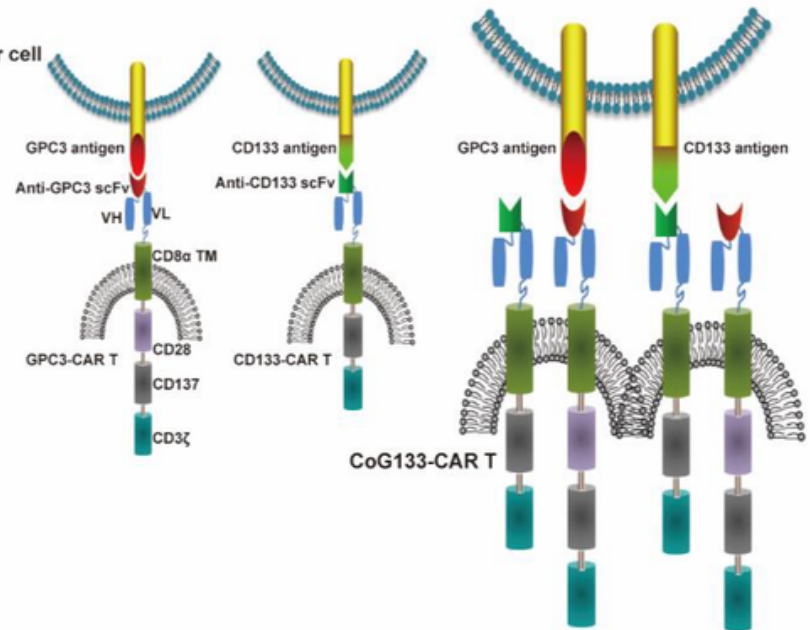

\section{Figure 1}

Construction and characterization of two mcDNA vectors and the structure of CoG133-CAR T cells. A) Schematic representation of CD133-CAR and GPC3-CAR construction. B) The double restriction enzyme digestion for selecting CAR structures. Lanes 1-3 contain three GPC3-CAR-positive bacterial clones, and lanes 4-6 contain three CD133-CAR-positive bacterial clones. M=molecular weight marker. C) Schematic diagram showing the generation of the CD133-CAR mcDNA and GPC3-CAR mcDNA. D) Electrophoretic 
analysis to detect mcDNA. After L-arabinose induction, CD133-CAR mcDNA and GPC3-CAR mcDNA were digested into fragments of $5179 \mathrm{bp}$ and $4608 \mathrm{bp}$, respectively. E) Schematic illustration of the CoG133CAR T cell structure. CD133-CAR T or GPC3-CAR T cells recognized only one tumor cell surface antigen, but CoG133-CAR T cells exerted a destructive effect on tumors containing CD133 and GPC3 antigens by recognizing one of those antigens.

A

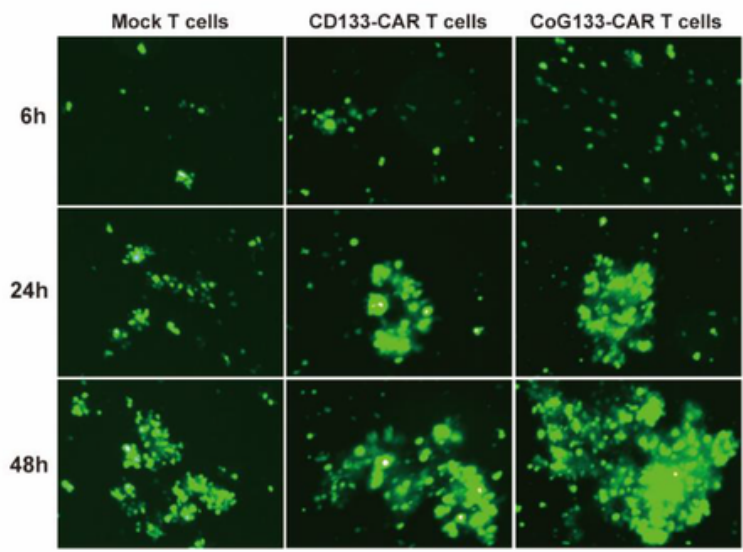

B
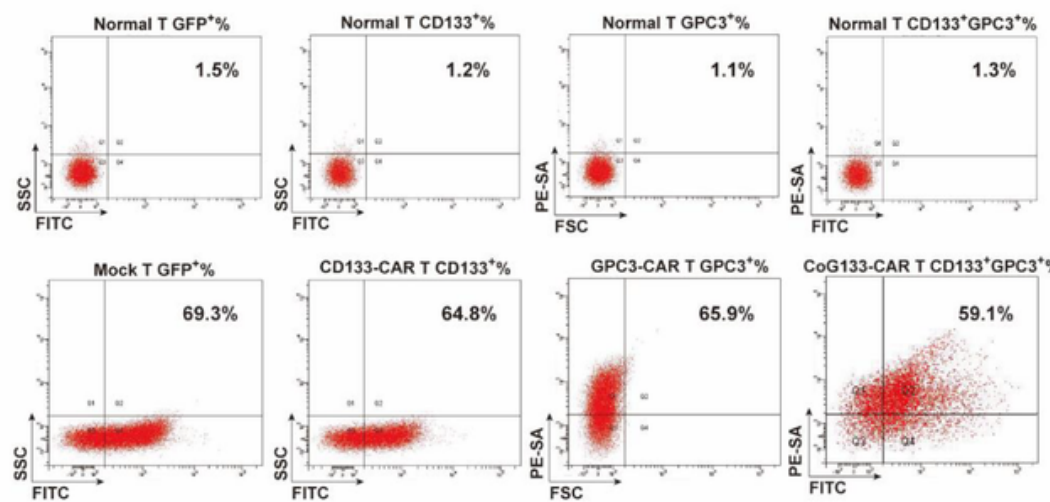

C
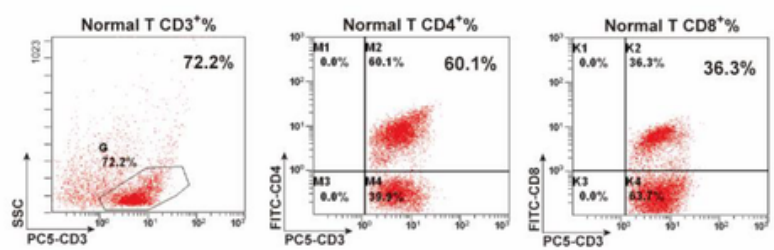

D
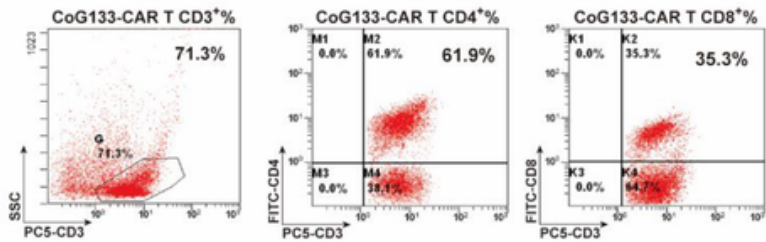

E
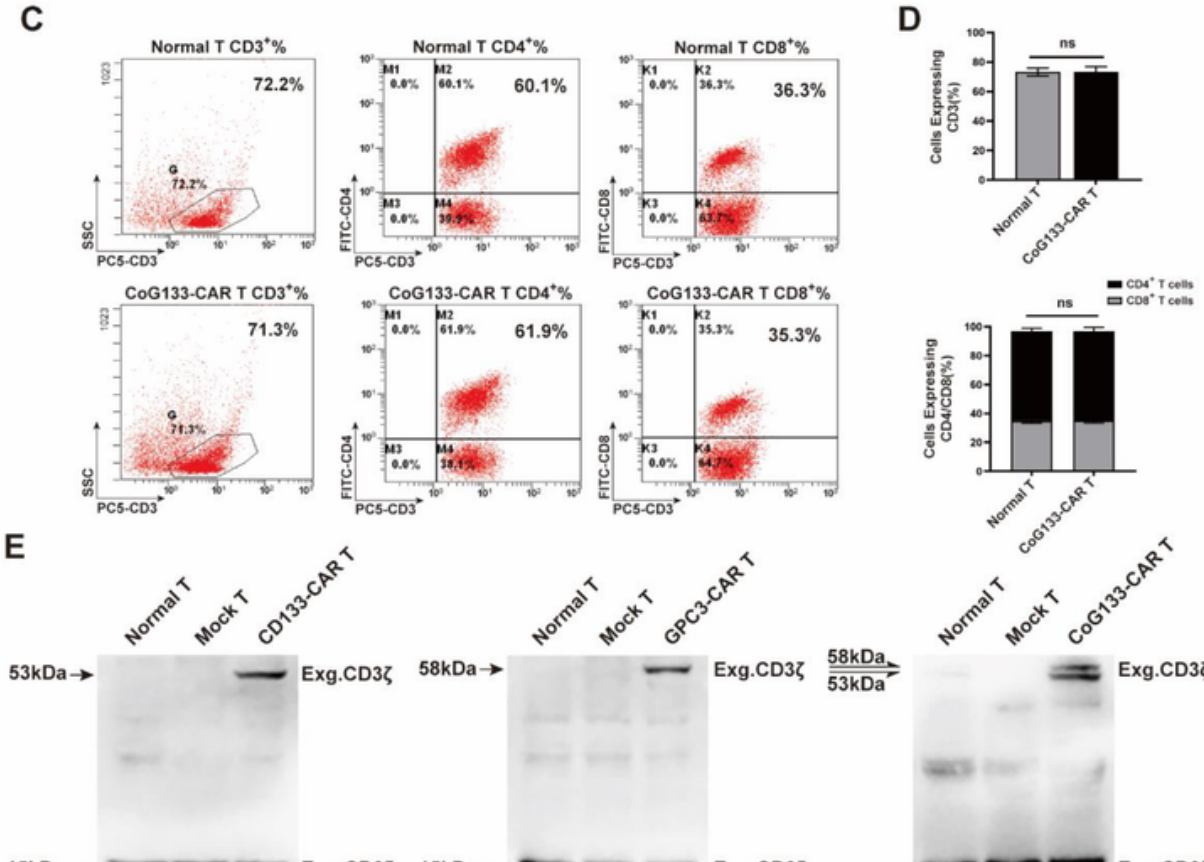

$15 \mathrm{kDa} \rightarrow$

Eng.CD3 $\zeta \quad 15 \mathrm{kDa} \rightarrow$

Eng.CD3

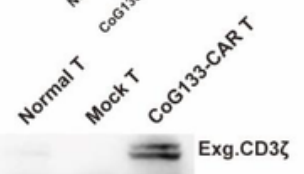

Figure 2 
Transfection efficiency and phenotype evaluation of CAR T cells, along with protein expression analysis. A) Fluorescence microscopy images of human T lymphocytes transfected with the mcDNA or plasmid encoding GFP. The expression of GFP was gradually increased in mock T cells, CD133-CAR T cells and CoG133-CAR T cells 6, 24 and 48 hours after electroporation at 400xmagnification. B) Flow cytometric analysis of CD133-CAR and GPC3-CAR expression in CAR T cells. Seven days after electroporation, the transfection efficiency of mock T, CD133-CAR T, GPC3-CAR T, and CoG133-CAR T cells was $69.3 \%, 64.8 \%$, $65.9 \%$, and $59.1 \%$, respectively. C) Flow cytometric analysis showed similar expression levels of CD3, CD 4 and CD8 on normal T and CoG133-CAR T cells 7 days after electroporation. D) Graph of the normal T and CoG133-CAR T cell phenotype analysis. Statistics are presented as the means \pm SDs. $n=3$ per group. E) Determination of CAR protein expression after transfection by Western blot analysis. The exogenous $\mathrm{CD} 3 \zeta$ protein was detected by chemiluminescence reagents to assess CAR protein expression. The molecular weights of the CD133-CAR and GPC3-CAR proteins were $53 \mathrm{kDa}$ and $58 \mathrm{kDa}$, and the lysates of CoG133-CAR T cells contained both proteins. 
A
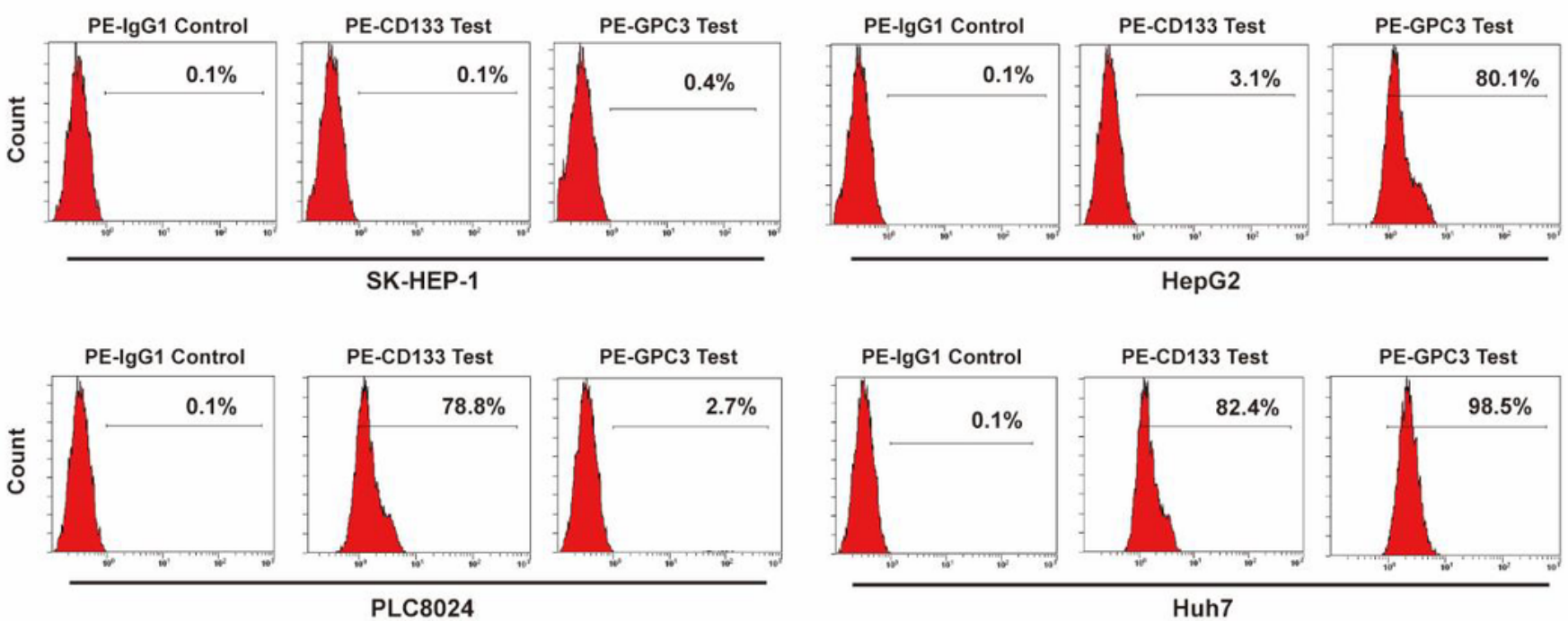

B

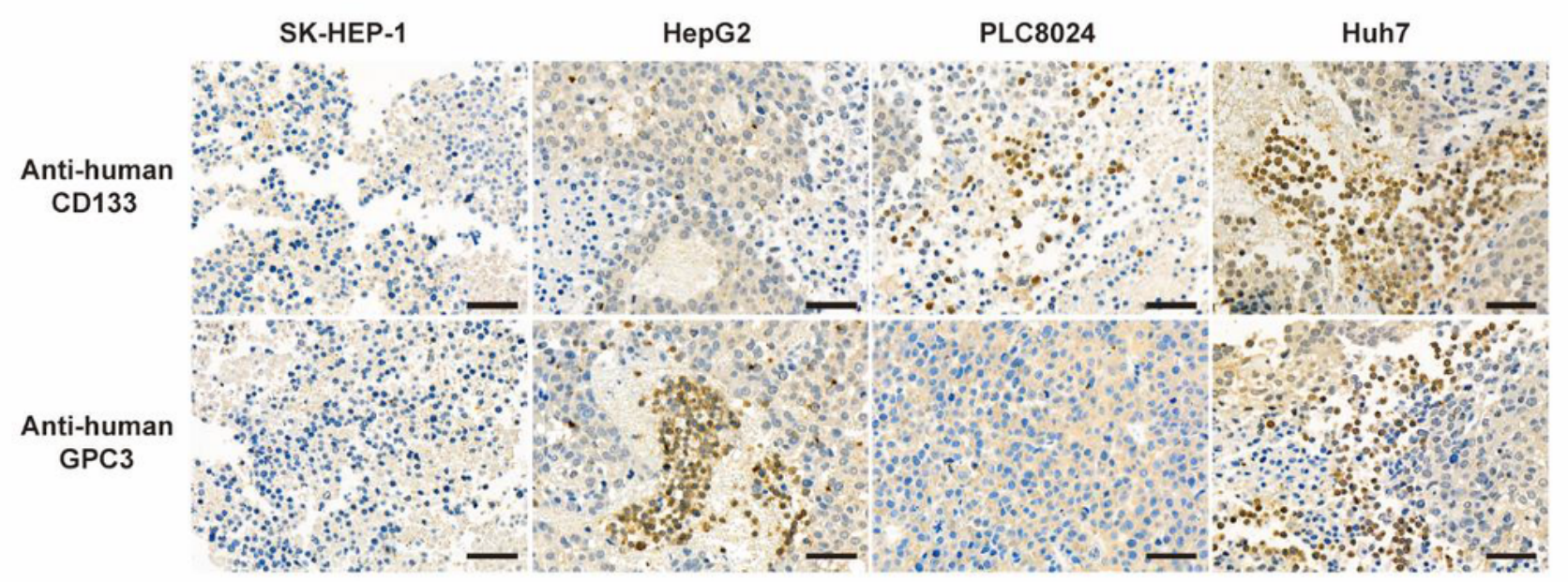

C

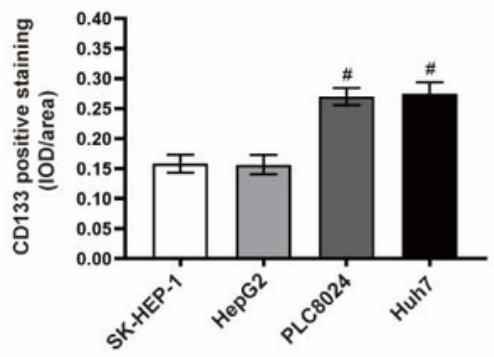

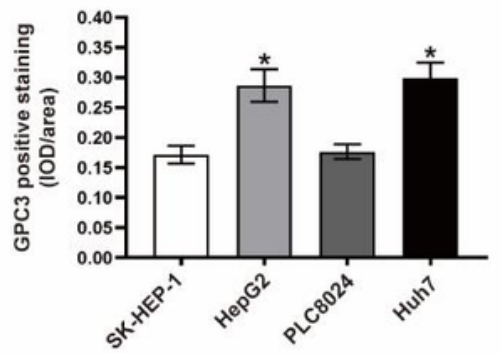

D

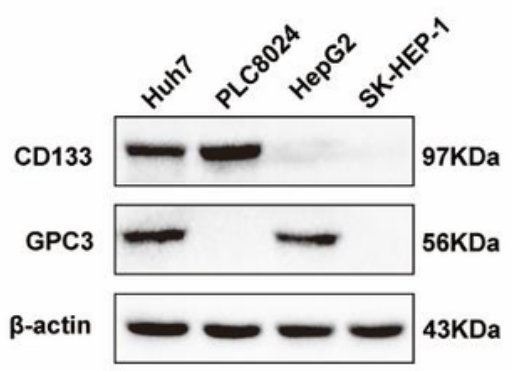

\section{Figure 3}

Analysis of CD133 and GPC3 expression in HCC. A) Flow cytometric analysis of CD133 and GPC3 antigen expression on the surface of cells from 4 human HCC cell lines. Tumor cells were stained with the PE-conjugated anti-IgG1 antibody (isotype control), PE-conjugated anti-CD133 mAb and PE-conjugated anti-GPC3 antibody. B) Representative immunohistochemical staining images showing CD133 and GPC3 antigen expression in human HCC tissues from NOD/SCID xenograft mice. Scale bar=50 $\mu \mathrm{m} . \mathrm{C}$ ) 
Semiquantitative IOD analysis of CD133+ and GPC3+ staining in human HCC cells. Statistics are presented as the means \pm SDs. $n=3$ per group. \#, $P<0.001$ vs. the SK-HEP-1 and HepG2 groups. *, $P<0.001$ vs. the SK-HEP-1 and PLC8024 groups. D) Western blot analysis showing the expression of CD133 and GPC3 proteins in human HCC tissues extracted from NOD/SCID xenograft mice inoculated with SK-HEP1, HepG2, PLC8024 and Huh7 cells.

A

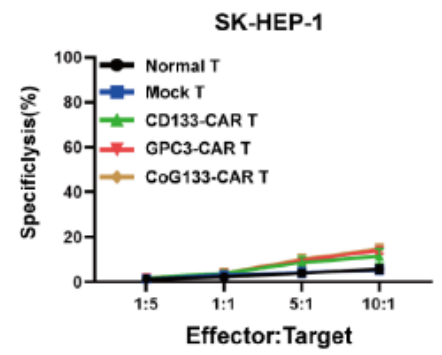

PLC8024

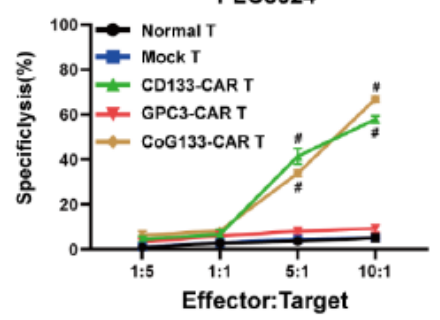

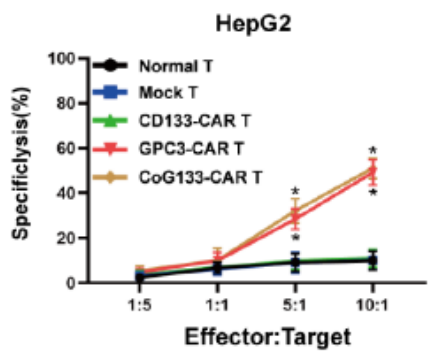

Huh7

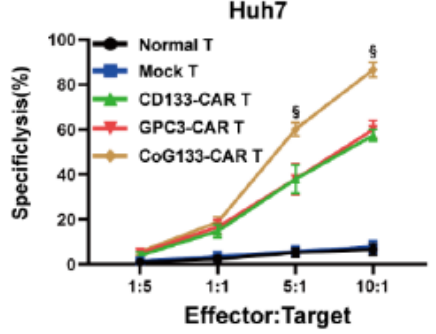

B
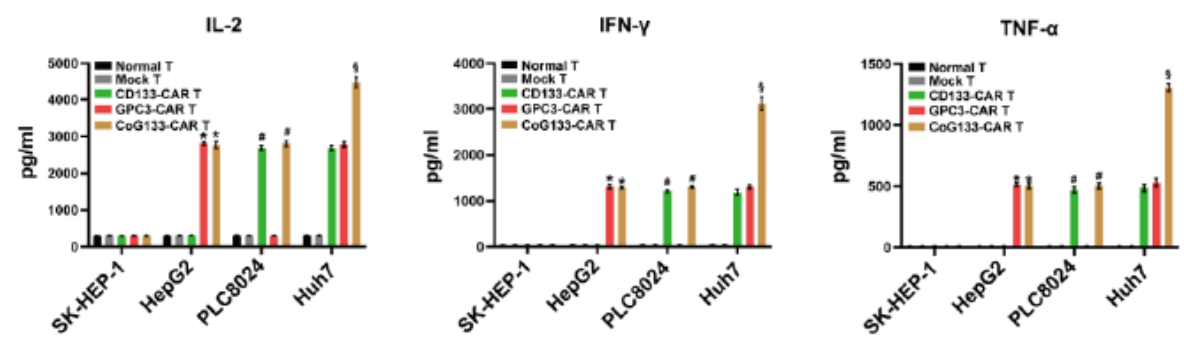

C
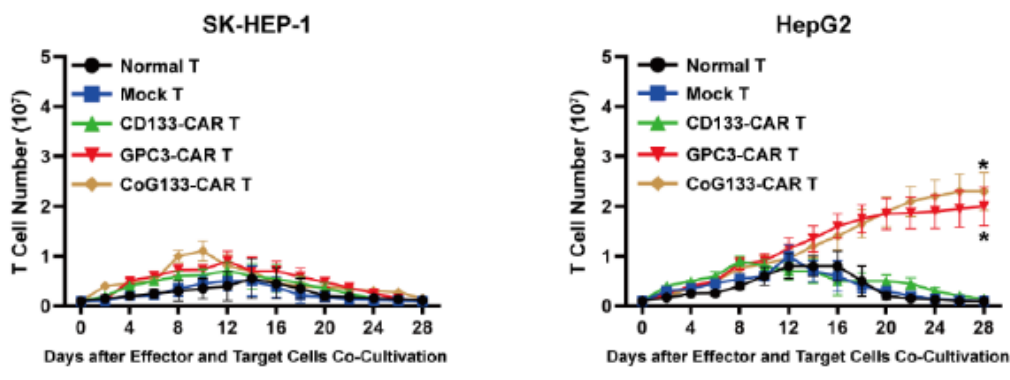

PLC8024
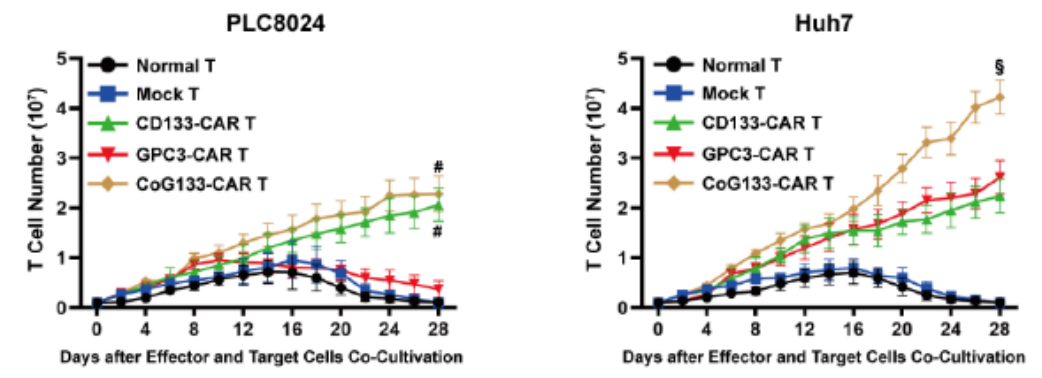

Figure 4 
Cytotoxicity activity, cytokine secretion and proliferation of CAR-engineered T cells in vitro. A) Normal T, mock $T$ and CAR-engineered T cells were co-incubated with human hepatocellular carcinoma cell lines for 18 hours at different Effector: Target ratios. B) ELISA analysis showed the secretion of IL-2, IFN- $y$ and TNF-a by Normal T, mock $T$ and CAR-engineered T cells which were co-incubated with tumor cells at a 1:1 Effector: Target ratio for $24 \mathrm{~h}$. C) We prepared 5×106 normal T, mock T and CAR-engineered T cells to cocultivate with human hepatocellular carcinoma cell lines for 28 days. We measured viable $T$ cell numbers every other day to reflect the proliferation of effector cells. All statistics were presented as mean $\pm S D . n=$ 6 in each group, ${ }^{*}, \mathrm{P}<0.001$ vs. normal T, mock T and CD133-CAR T groups; \#, $\mathrm{P}<0.001$ vs. normal T, mock T and GPC3-CAR T groups; §, P<0.001 vs. normal T, mock T, CD133 CAR-T and GPC3-CAR T groups. 
A

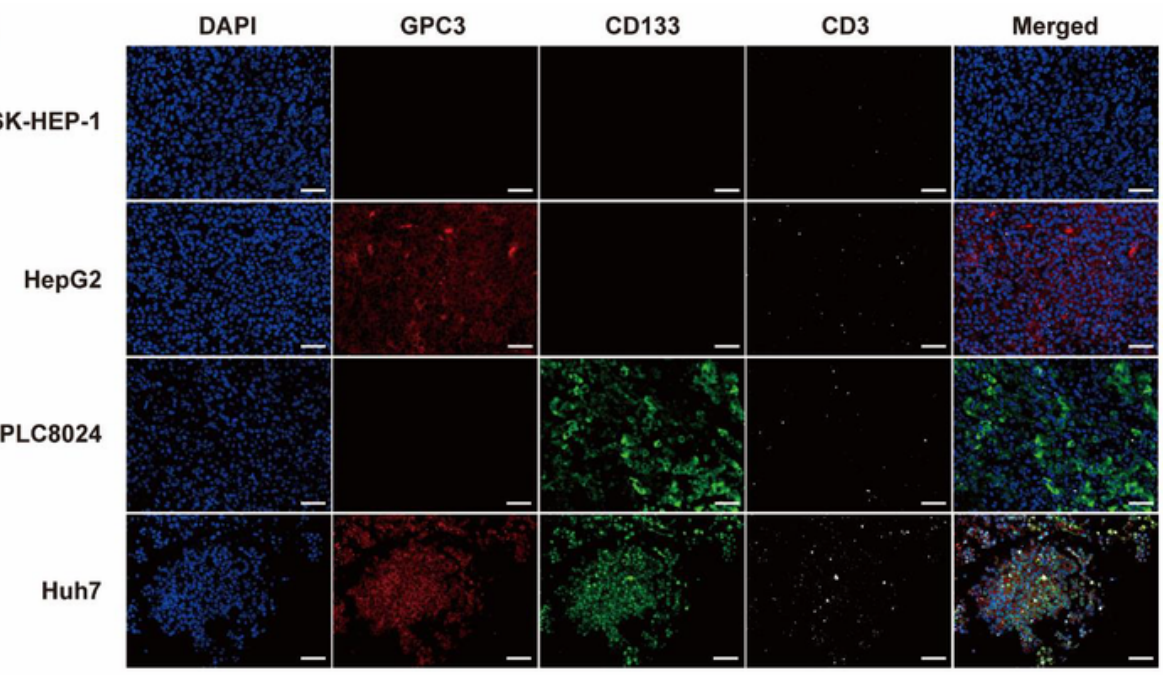

B

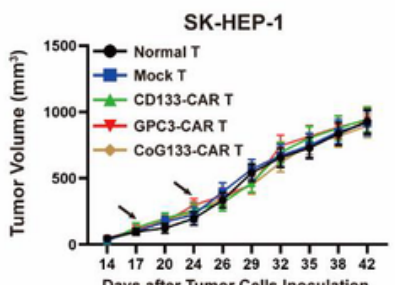

HepG2
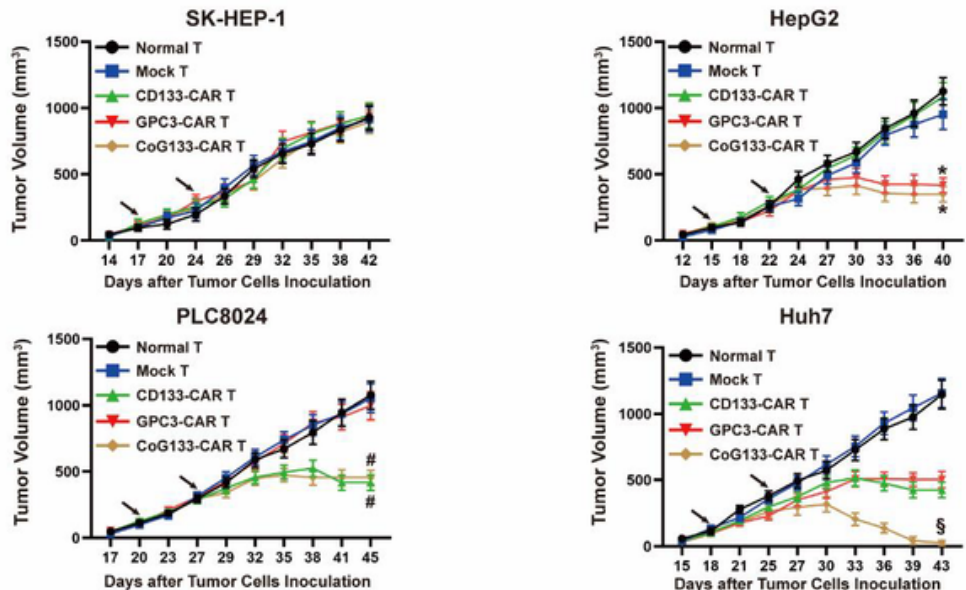

Huh7

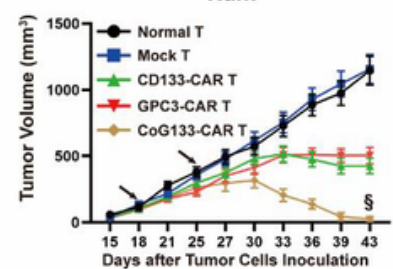

C
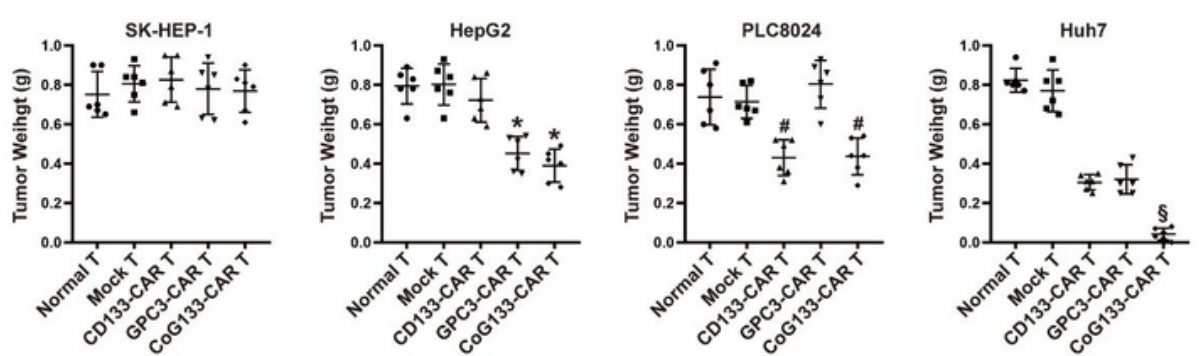

IL-2

IFN-Y
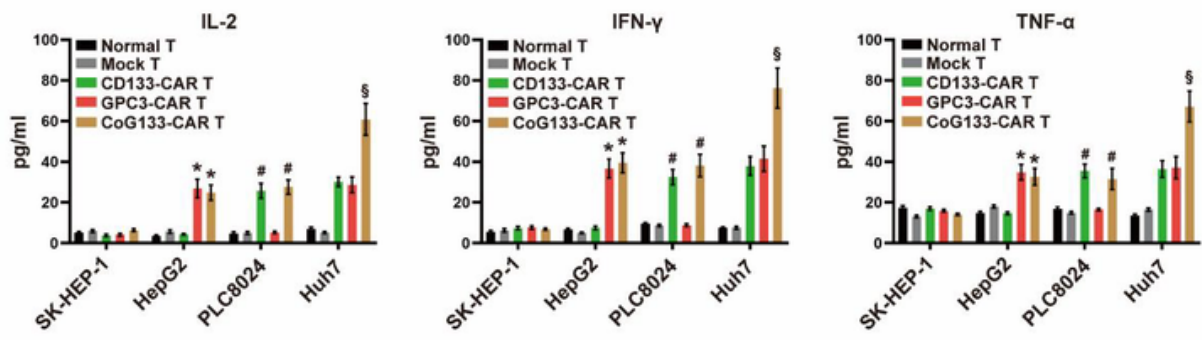

\section{Figure 5}

In vivo inhibitory effect of CAR-engineered T cells on tumors. A) Representative immunofluorescence images of 4 tumor tissue sections from NOD/SCID xenograft mice injected with CoG133-CAR T cells (sacrificed on day 29 after tumor formation). CD133 is labeled in green, GPC3 is labeled in red and exogenous CD3 is labeled in white; scale bar $=50 \mu \mathrm{m}$. B) We injected effector cells into NOD/SCID xenograft mice on day 0 and day 7 (arrows marked) after the tumor volume was approximately $100 \mathrm{~mm} 3$ 
and recorded the tumor volume data. C) We measured the weight of tumor tissues isolated from the sacrificed mice. D) ELISA showing cytokine secretion in mouse blood serum 7 days after treatment. All statistics are presented as the means $\pm S D s . n=6$ per group. * $\mathrm{P}<0.001$ vs. the normal $T$, mock $T$ and CD133-CAR T groups; \#, P<0.001 vs. the normal T, mock T and GPC3-CAR T groups; $§, P<0.001$ vs. the normal T, mock T, CD133-CAR T and GPC3-CAR T groups.

A

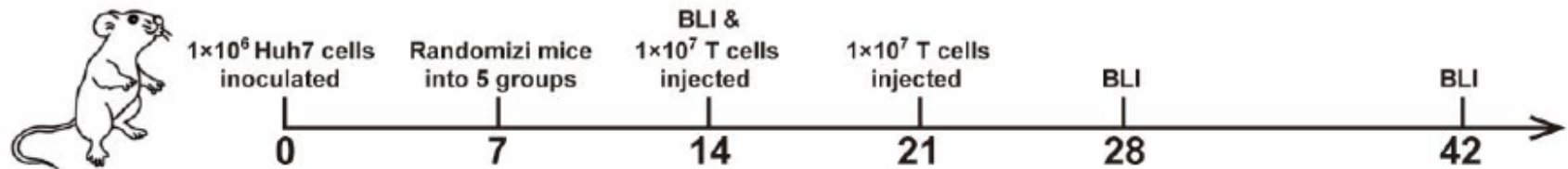

B

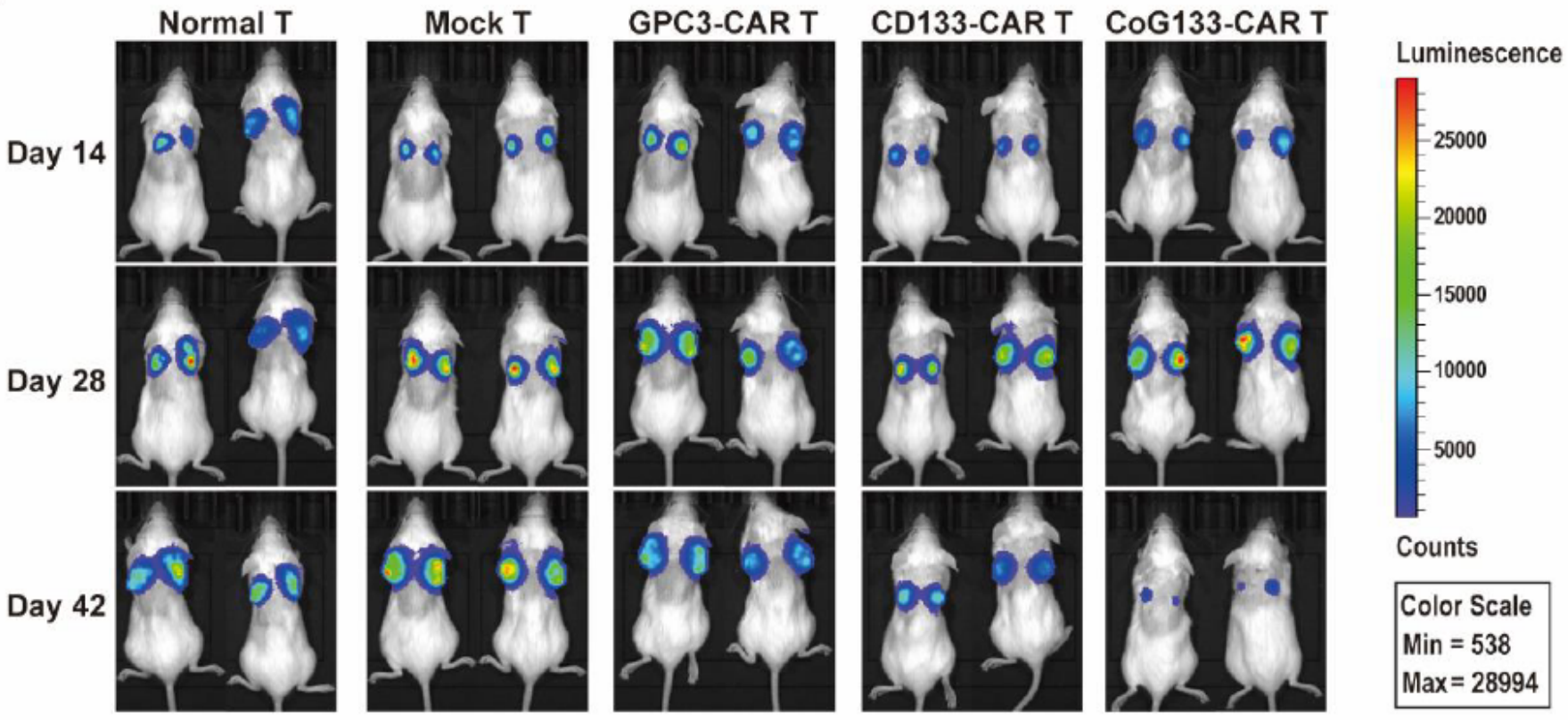

C

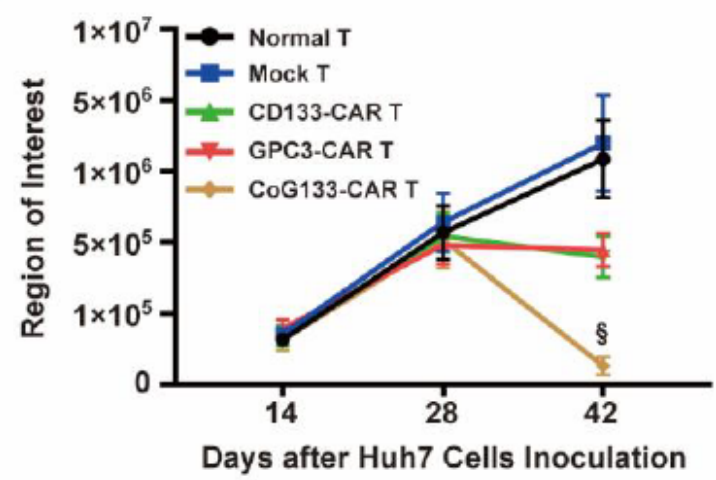

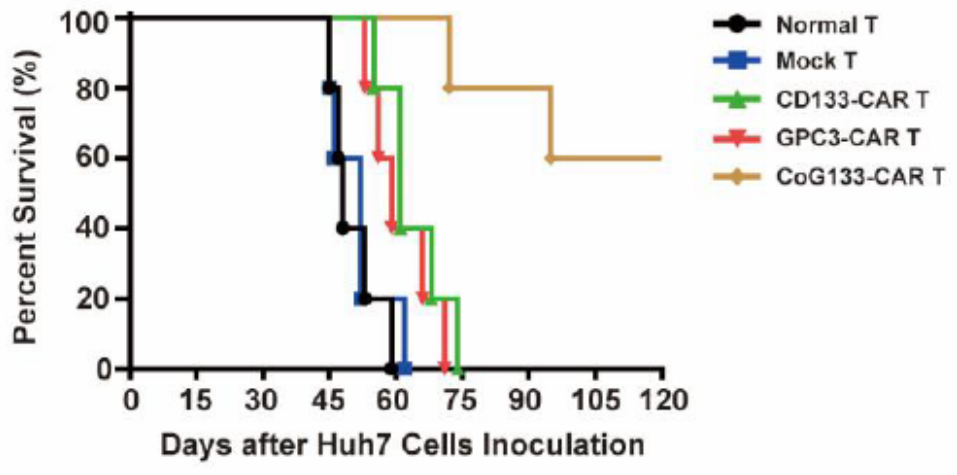

Figure 6 
Antitumor efficacy of CoG133-CAR T cells in xenograft mice bearing double-positive tumors. A) Schematic representation of the in vivo mouse bioluminescence study. B) The tumor-derived bioluminescence images of mice inoculated with Huh7 cells and treated with normal T and CoG133-CAR T cells. C) Statistical analysis of the bioluminescence images in the ROI at each time point. Statistics are presented as the means $\pm S D s . n=5$ per group. $\S, P<0.001$ vs. the normal $T$, mock $T, C D 133-C A R T$ and GPC3-CAR T groups. D) Survival curve showed the survival time of Huh7 tumor bearing mice treated with different effector cells. The results were evaluated by the log-rank (Mantel-Cox) test. $n=5$ per group. Pvalue 0.001 . 
A
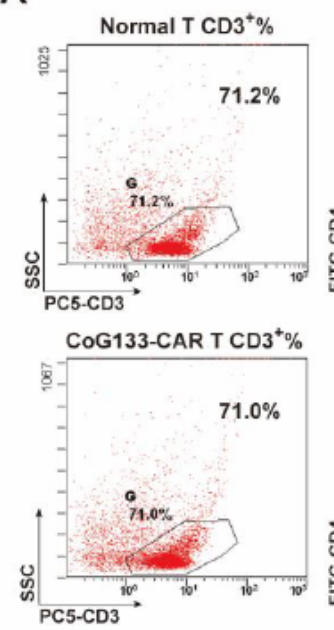

C

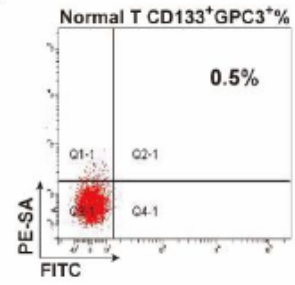

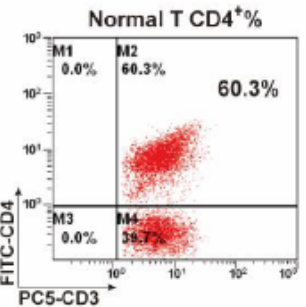

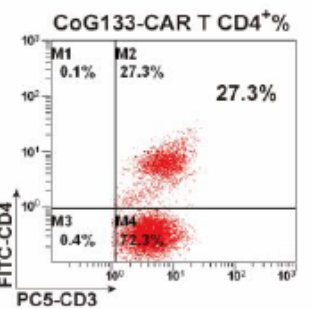

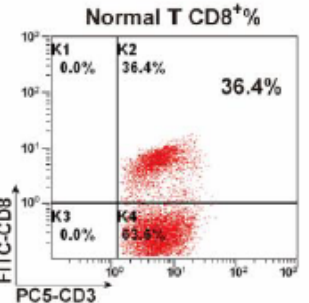

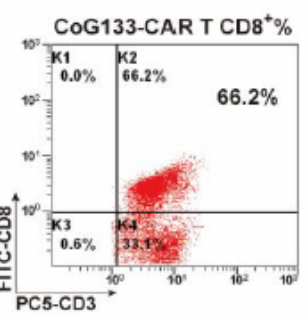

D

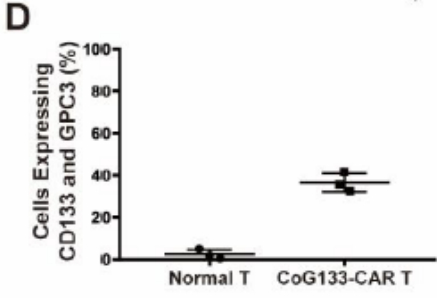

B

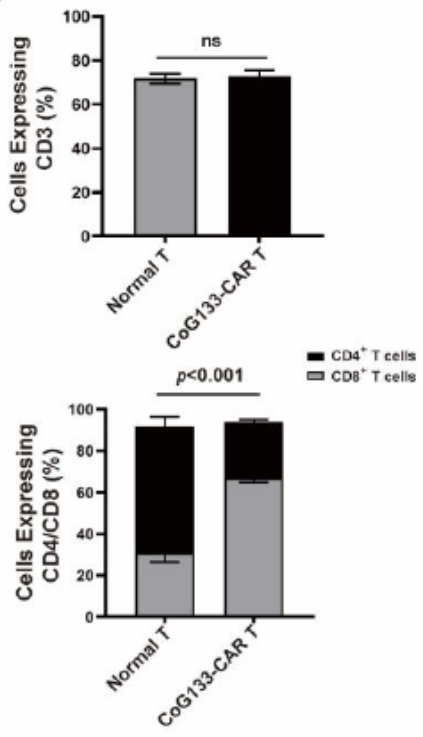

E
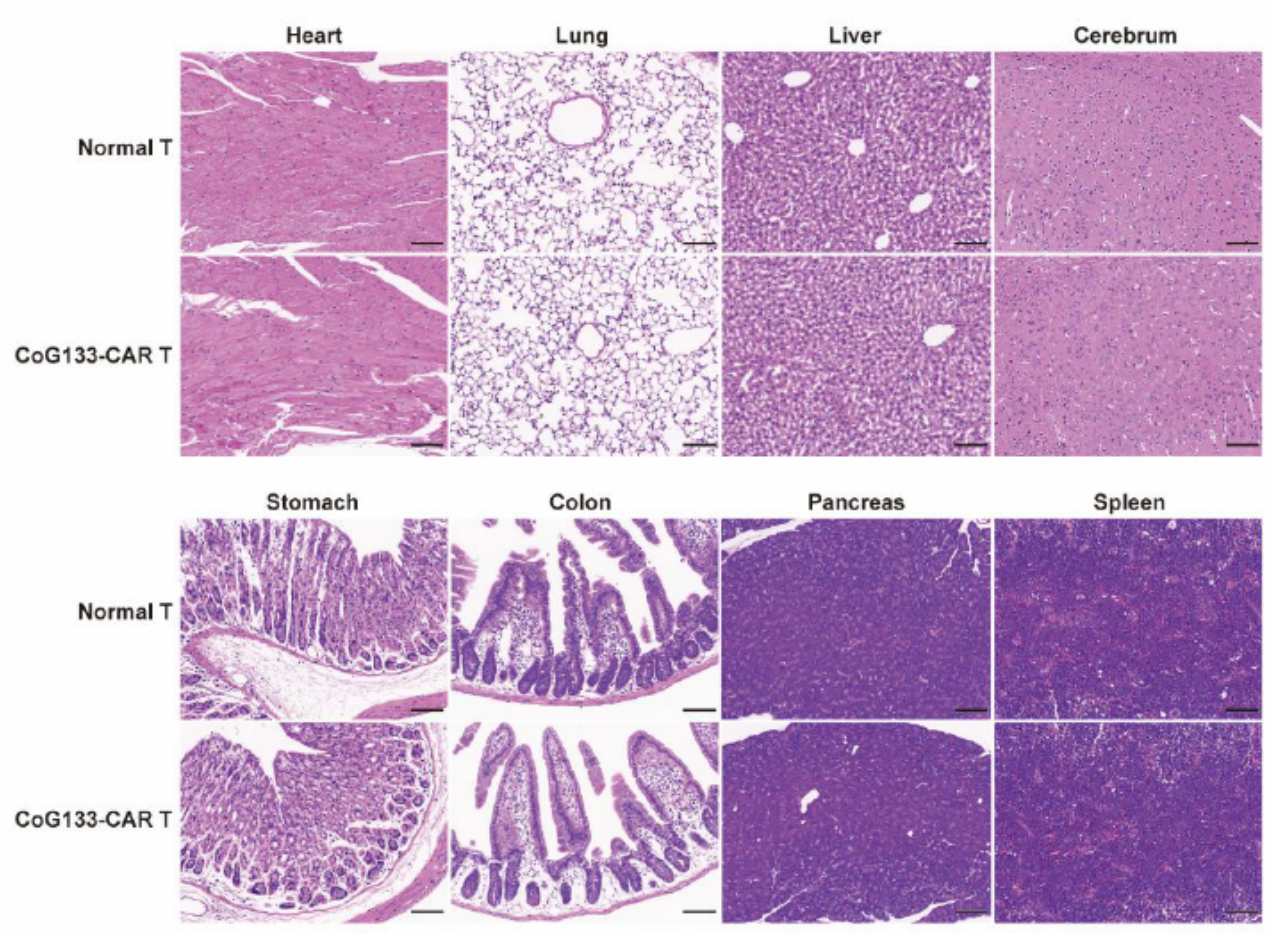

\section{Figure 7}

Phenotype, persistence and safety of CoG133-CAR T cells. A) Flow cytometric analysis showed the phenotype of CAR T and normal T cells from the peripheral blood of Huh7 xenograft mice on day 7 . B) Statistical analysis showed the proportion of CD3+, CD4+ and CD8+ normal T and CoG133-CAR T cells. Statistics are presented as the means \pm SDs. $n=3$ per group. C) CD133 and GPC3 coexpression was detected by flow cytometry. D) Statistical analysis showed the CoG133-CAR T cells expression. Statistics 
are presented as the means \pm SDs. $n=3$ per group. E) We harvested murine organ tissues from 4 groups of tumor xenograft mice sacrificed 28 days after the second injection of effector cells. The tissues were stained with H\&E. The histopathological images of the organ tissues from all groups of tumor-bearing mice showed no differences, and images from the Huh7 group are shown as a representative. Scale bar $=100 \mu \mathrm{m}$. 